\title{
Pacific
}

Journal of

Mathematics

\section{COACTION FUNCTORS}

S. KaLiszewski, Magnus B. LANDSTAD AND John QUigG 


\title{
COACTION FUNCTORS
}

\section{S. KALISZEWSKI, MAGnus B. LANDSTAD AND JOHN QUIGG}

\begin{abstract}
A certain type of functor on a category of coactions of a locally compact group on $C^{*}$-algebras is introduced and studied. These functors are intended to help in the study of the crossed-product functors that have been recently introduced in relation to the Baum-Connes conjecture. The most important coaction functors are the ones induced by large ideals of the Fourier-Stieltjes algebra. It is left as an open problem whether the "minimal exact and Morita compatible crossed-product functor" is induced by a large ideal.
\end{abstract}

\section{Introduction}

In [Baum et al. 2016], with an eye toward expanding the class of locally compact groups $G$ for which the Baum-Connes conjecture holds, the authors study "crossedproduct functors" that take an action of $G$ on a $C^{*}$-algebra and produce an "exotic crossed product" between the full and reduced ones, in a functorial manner.

In [KLQ 2013], inspired by [Brown and Guentner 2013], we studied certain quotients of $C^{*}(G)$ that lie "above" $C_{r}^{*}(G)$ - namely those that carry a quotient coaction. We characterized these intermediate (which we now call "large") quotients as those for which the annihilator $E$, in the Fourier-Stieltjes algebra $B(G)$, of the kernel of the quotient map is a $G$-invariant weak*-closed ideal containing the reduced Fourier-Stieltjes algebra $B_{r}(G)$ (which we now call "large ideals" of $B(G)$ ). We went on to show how, if $\alpha$ is an action of $G$ on a $C^{*}$-algebra $B$, large ideals $E$ induce exotic crossed products $B \rtimes_{\alpha, E} G$ intermediate between the full and reduced crossed products $B \rtimes_{\alpha} G$ and $B \rtimes_{\alpha, r} G$. One of the reasons this interested us is the possibility of " $E$-crossed-product duality" for a coaction $\delta$ of $G$ on a $C^{*}$-algebra $A$ : namely, that the canonical surjection

$$
\Phi: A \rtimes_{\delta} G \rtimes_{\widehat{\delta}} G \rightarrow A \otimes \mathcal{K}\left(L^{2}(G)\right)
$$

descends to an isomorphism

$$
A \rtimes_{\delta} G \rtimes_{\widehat{\delta}, E} G \cong A \otimes \mathcal{K} .
$$

MSC2010: primary 46L55; secondary 46M15.

Keywords: crossed product, action, coaction, Fourier-Stieltjes algebra, exact sequence, Morita compatible. 
Crossed-product duality

$$
A \rtimes_{\delta} G \rtimes_{\widehat{\delta}, r} G \cong A \otimes \mathcal{K}
$$

for normal coactions and

$$
A \rtimes_{\delta} G \rtimes_{\widehat{\delta}} G \cong A \otimes \mathcal{K}
$$

for maximal coactions are the extreme cases with $E=B_{r}(G)$ and $B(G)$, respectively. We (rashly) conjectured that every coaction satisfies $E$-crossed-product duality for some $E$, and moreover that the dual coaction on every $E$-crossed product $B \rtimes_{\alpha, E} G$ satisfies $E$-crossed-product duality.

Buss and Echterhoff [2014] disproved the first of the above conjectures and proved the second, and in [KLQ 2016] we independently proved the second conjecture. (Note: in that paper we wrote "We originally wondered whether every coaction satisfies $E$-crossed product duality for some $E$. In [KLQ 2013, Conjecture 6.12] we even conjectured that this would be true for dual coactions." This is slightly inaccurate - [KLQ 2013, Conjecture 6.14] concerns dual coactions, while Conjecture 6.12 says "Every coaction satisfies $E$-crossed-product duality for some $E$.")

In [KLQ 2016, Section 3] we showed that every large ideal $E$ of $B(G)$ induces a transformation $(A, \delta) \mapsto\left(A^{E}, \delta^{E}\right)$ of $G$-coactions, where $A^{E}=A / A_{E}$ and $A_{E}=\operatorname{ker}\left(\mathrm{id} \otimes q_{E}\right) \circ \delta$, and where in turn

$$
q_{E}: C^{*}(G) \rightarrow C_{E}^{*}(G):=C^{*}(G) /^{\perp} E
$$

is the quotient map.

In this paper we further study this assignment $(A, \delta) \mapsto\left(A^{E}, \delta^{E}\right)$. When $(A, \delta)=$ $\left(B \rtimes_{\alpha} G, \hat{\alpha}\right)$, the composition

$$
(B, \alpha) \mapsto\left(B \rtimes_{\alpha} G, \widehat{\alpha}\right) \mapsto\left(B \rtimes_{\alpha, E} G, \hat{\alpha}^{E}\right)
$$

was shown to be functorial in [Buss and Echterhoff 2014, Corollary 6.5]; here we show that $(A, \delta) \mapsto\left(A^{E}, \delta^{E}\right)$ is functorial, giving an alternate proof of the Buss-Echterhoff result.

In fact, we study more general functors on the category of coactions of $G$, of which the functors induced by large ideals of $B(G)$ are special cases. We are most interested in the connection with the crossed-product functors of [Baum et al. 2016]. In particular, we introduce a "minimal exact and Morita compatible" coaction functor. When this functor is composed with the full-crossed-product functor for actions, the result is a crossed-product functor in the sense of [loc. cit.]. We briefly discuss various possibilities for how these functors are related: for example, is the composition mentioned in the preceding sentence equal to the minimal exact and Morita compatible crossed-product functor of [loc. cit.]? Also, is the greatest lower bound of the coaction functors defined by large ideals itself defined by a large ideal? 
These are just two among others that arise naturally from these considerations. Unfortunately, at this early stage we have more questions than answers.

After a short section on preliminaries, in Section 3 we define the categories we will use for our functors. In numerous previous papers, we have used "nondegenerate categories" of $C^{*}$-algebras and their equivariant counterparts. But these categories are inappropriate for the current paper, primarily due to our need for short exact sequences. Rather, here we must use "classical" categories, where the homomorphisms go between the $C^{*}$-algebras themselves, not into multiplier algebras. In order to avail ourselves of tools that have been developed for the equivariant nondegenerate categories, we include a brief summary of how the basic theory works for the classical categories. Interestingly, the crossed products are the same in both versions of the categories (see Corollaries 3.9 and 3.13).

In Section 4 we define coaction functors, which are a special type of functor on the classical category of coactions. Composing such a coaction functor with the full-crossed-product functor on actions, we get crossed-product functors in the sense of Baum, Guentner and Willett [loc. cit.]; it remains an open problem whether every such crossed-product functor is of this form. Maximalization and normalization are examples of coaction functors, but there are lots more - for example, the functors induced by large ideals of the Fourier-Stieltjes algebra (see Section 6). In Section 4 we also define a partial ordering on coaction functors, and prove in Theorem 4.9 that the class of coaction functors is complete in the sense that every nonempty collection of them has a greatest lower bound. We also introduce the general notions of exact or Morita compatible coaction functors, and prove in Theorem 4.22 that they are preserved by greatest lower bounds. We show in Proposition 4.24 that our partial order, exactness and Morita compatibility are consistent with those of [loc. cit.].

To help prepare for the study of coaction functors associated to large ideals, in Section 5 we introduce decreasing coaction functors, and show how Morita compatibility takes a particularly simple form for these functors in Proposition 5.5.

In Section 6 we study the coaction functors $\tau_{E}$ induced by large ideals $E$ of $B(G)$. Perhaps interestingly, maximalization is not among these functors. We show that these functors $\tau_{E}$ are decreasing in Proposition 6.2, and how the test for exactness simplifies significantly for them in Proposition 6.7. Moreover, $\tau_{E}$ is automatically Morita compatible (see Proposition 6.10). Composing maximalization followed by $\tau_{E}$, we get a related functor that we call $E$-ization. We show that these functors are also Morita compatible in Theorem 6.14. Although $E$-ization and $\tau_{E}$ have similar properties, they are not naturally isomorphic functors (see Remark 6.15). The outputs of $E$-ization are precisely the coactions we call $E$-coactions, namely those for which $E$-crossed-product duality holds [KLQ 2016, Theorem 4.6] (see also [Buss and Echterhoff 2014, Theorem 5.1]). Theorem 6.17 shows that $\tau_{E}$ gives 
an equivalence of maximal coactions with $E$-coactions. We close Section 6 with some open problems that mainly concern the application of the coaction functors $\tau_{E}$ to the theory of [Baum et al. 2016].

Finally, the Appendix supplies a few tools that show how some properties of coactions can be more easily handled using the associated $B(G)$-module structure.

\section{Preliminaries}

We refer to [Echterhoff et al. 2004; 2006, Appendix A] for background material on coactions of locally compact groups on $C^{*}$-algebras, and [Echterhoff et al. 2006, Chapters 1-2] for imprimitivity bimodules and their linking algebras. Throughout, $G$ will denote a locally compact group, and $A, B, C, \ldots$ will denote $C^{*}$-algebras.

Recall from [loc. cit., Definition 1.14] that the multiplier bimodule of an $A-B$ imprimitivity bimodule $X$ is defined as $M(X)=\mathcal{L}_{B}(B, X)$, where $B$ is regarded as a Hilbert module over itself in the canonical way. Also recall [loc. cit., Corollary 1.13] that $M(X)$ becomes an $M(A)-M(B)$ correspondence in a natural way. The linking algebra of an $A-B$ imprimitivity bimodule $X$ is

$$
L(X)=\left(\begin{array}{ll}
A & X \\
\tilde{X} & B
\end{array}\right)
$$

where $\tilde{X}$ is the dual $B-A$ imprimitivity bimodule. $A, B$ and $X$ are recovered from $L(X)$ via the corner projections

$$
p=\left(\begin{array}{ll}
1 & 0 \\
0 & 0
\end{array}\right), q=\left(\begin{array}{ll}
0 & 0 \\
0 & 1
\end{array}\right) \in M(L(X)) .
$$

The multiplier algebra of $L(X)$ decomposes as

$$
M(L(X))=\left(\begin{array}{ll}
M(A) & M(X) \\
M(\tilde{X}) & M(B)
\end{array}\right)
$$

We usually omit the lower left corner of the linking algebra, writing $L(X)=\left(\begin{array}{ll}A & X \\ * & B\end{array}\right)$, since it takes care of itself. Also recall from [loc. cit., Lemma 1.52] (see also [Echterhoff and Raeburn 1995, Remark (2), p. 307]) that nondegenerate homomorphisms of imprimitivity bimodules correspond bijectively to nondegenerate homomorphisms of their linking algebras.

For an action $(A, \alpha)$ of $G$, we use the following notation for the (full) crossed product $A \rtimes_{\alpha} G$ :

- $i_{A}=i_{A}^{\alpha}: A \rightarrow M\left(A \rtimes_{\alpha} G\right)$ and $i_{G}=i_{G}^{\alpha}: G \rightarrow M\left(A \rtimes_{\alpha} G\right)$ make up the universal covariant homomorphism $\left(i_{A}, i_{G}\right)$.

- $\hat{\alpha}$ is the dual coaction on $A \rtimes_{\alpha} G$. 
On the other hand, for the reduced crossed product $A \rtimes_{\alpha, r} G$ we use the following notation:

- $\Lambda: A \rtimes_{\alpha} G \rightarrow A \rtimes_{\alpha, r} G$ is the regular representation.

- $i_{A}^{r}=i_{A}^{\alpha, r}=\Lambda \circ i_{A}$ and $i_{G}^{r}=i_{G}^{\alpha, r}=\Lambda \circ i_{G}$ are the canonical maps into $M\left(A \rtimes_{\alpha, r} G\right)$.

- $\widehat{\alpha}^{n}$ is the dual coaction on $A \rtimes_{\alpha, r} G$.

We will need to work extensively with morphisms between coactions, in particular (but certainly not only) with maximalization and normalization. In the literature, the notation for these maps has not yet stabilized. Recall that a coaction $(A, \delta)$ is called normal if the canonical surjection

$$
\Phi: A \rtimes_{\delta} G \rtimes_{\widehat{\delta}} G \rightarrow A \otimes \mathcal{K}\left(L^{2}(G)\right)
$$

factors through an isomorphism of the reduced crossed product

$$
\Phi_{r}: A \rtimes_{\delta} G \rtimes_{\widehat{\delta}, r} G \rightarrow A \otimes \mathcal{K}\left(L^{2}(G)\right),
$$

and maximal if $\Phi$ itself is an isomorphism. One convention is, for a coaction $(A, \delta)$ of $G$, to write

$$
q_{A}^{m}:\left(A^{m}, \delta^{m}\right) \rightarrow(A, \delta)
$$

for a maximalization, and

$$
q_{A}^{n}:(A, \delta) \rightarrow\left(A^{n}, \delta^{n}\right)
$$

for a normalization. We will use this convention for maximalization, but we will need the letter " $q$ " for other similar purposes, and it would be confusing to keep using it for normalization. Instead, we will use

$$
\Lambda=\Lambda_{A}:(A, \delta) \rightarrow\left(A^{n}, \delta^{n}\right)
$$

for normalization - this is supposed to remind us that for crossed products by actions the regular representation

$$
\Lambda:\left(A \rtimes_{\alpha} G, \widehat{\alpha}\right) \rightarrow\left(A \rtimes_{\alpha, r} G, \hat{\alpha}^{n}\right)
$$

is a normalization.

$\boldsymbol{B}(\boldsymbol{G})$-modules. Every coaction $(A, \delta)$ of $G$ induces $B(G)$-module structures on both $A$ and $A^{*}$ : for $f \in B(G)$, define

$$
\begin{array}{ll}
f \cdot a=(\mathrm{id} \otimes f) \circ \delta(a) & \text { for } a \in A, \\
(\omega \cdot f)(a)=\omega(f \cdot a) & \text { for } \omega \in A^{*}, a \in A .
\end{array}
$$


Many properties of coactions can be handled using these module structures rather than the coactions themselves. For example (see the Appendix), letting $(A, \delta)$ and $(B, \varepsilon)$ be coactions of $G$ :

(1) A homomorphism $\phi: A \rightarrow B$ is $\delta-\varepsilon$ equivariant, meaning $\varepsilon \circ \phi=\overline{\phi \otimes i d} \circ \delta$, if and only if

$$
\phi(f \cdot a)=f \cdot \phi(a) \text { for all } f \in B(G), a \in A .
$$

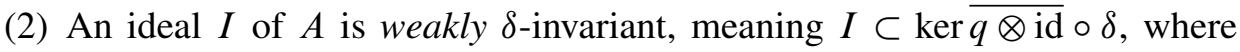
$q: A \rightarrow A / I$ is the quotient map, if and only if

$$
B(G) \cdot I \subset I,
$$

because the proof of [KLQ 2013, Lemma 3.11] shows that

$$
\operatorname{ker}(q \otimes \mathrm{id}) \circ \delta=\{a \in A: B(G) \cdot a \subset I\} .
$$

If $I$ is a weakly $\delta$-invariant ideal of $A$, then in fact $I=\operatorname{ker}(q \otimes$ id $) \circ \delta$, and the quotient map $q$ is $\delta-\delta^{I}$ equivariant for a unique coaction $\delta^{I}$ on $A / I$, which we call the quotient coaction. Since the slice map id $\otimes f: M\left(A \otimes C^{*}(G)\right) \rightarrow M(A)$ is strictly continuous [Landstad et al. 1987, Lemma 1.5], the $B(G)$-module structure extends to $M(A)$, and moreover $m \mapsto f \cdot m$ is strictly continuous on $M(A)$ for every $f \in B(G)$.

Short exact sequences. Several times we will need the following elementary lemma.

Lemma 2.1. Let

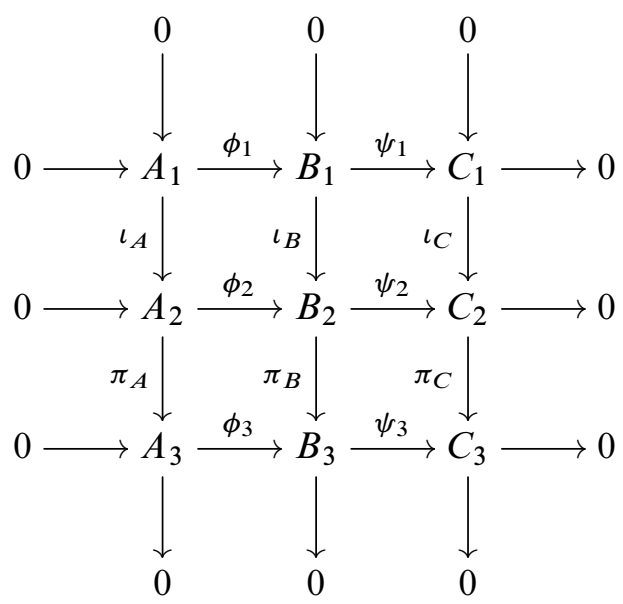

be a commutative diagram of $C^{*}$-algebras, where the columns and the middle row are exact. Suppose that the $\iota_{\bullet}$ are inclusions of ideals and the $\pi_{\bullet}$ are quotient maps. 
Then the bottom (interesting) row is exact if and only if both

$$
\phi_{2}\left(A_{1}\right)=\phi_{2}\left(A_{2}\right) \cap B_{1}
$$

and

$$
\phi_{2}\left(A_{2}\right)+B_{1} \supset \psi_{2}^{-1}\left(C_{1}\right) .
$$

Proof. Since $\psi_{3} \circ \pi_{B}=\pi_{C} \circ \psi_{2}$ and $\psi_{B}$ and $\phi_{2}$ are both surjective, $\psi_{3}$ is surjective, so the bottom row is automatically exact at $C_{3}$.

Thus, the only items to consider are exactness of the bottom row at $A_{3}$ and $B_{3}$, i.e., whether $\phi_{3}$ is injective and $\phi_{3}\left(A_{3}\right)=\operatorname{ker} \psi_{3}$.

The map $\phi_{3}$ is injective if and only if $\operatorname{ker} \pi_{A}=\operatorname{ker} \pi_{B} \circ \phi_{2}$, which, since $\phi_{2}$ is injective, is equivalent to (2-1).

Since $\psi_{2} \circ \phi_{2}=0$ and $\pi_{A}$ is surjective, $\psi_{3} \circ \phi_{3}=0$, so $\phi_{3}\left(A_{3}\right) \subset \operatorname{ker} \psi_{3}$ automatically. Since $\pi_{B}$ is surjective, $\phi_{3}\left(A_{3}\right) \supset \operatorname{ker} \psi_{3}$ if and only if

$$
\pi_{B}^{-1}\left(\phi_{3}\left(A_{3}\right) \supset \pi_{B}^{-1}\left(\operatorname{ker} \psi_{3}\right) .\right.
$$

Since $\pi_{B}^{-1}\left(\phi_{3}\left(A_{3}\right)\right)$ consists of all $b \in B_{2}$ for which

$$
\pi_{B}(a) \in \phi_{3}\left(A_{3}\right)=\phi_{3}\left(\pi_{A}\left(A_{2}\right)\right)=\pi_{B}\left(\phi_{2}\left(A_{2}\right)\right),
$$

equivalently for which

$$
b \in \phi_{2}\left(A_{2}\right)+B_{1},
$$

we see that

$$
\pi_{B}^{-1}\left(\phi_{3}\left(A_{3}\right)\right)=\phi_{2}\left(A_{2}\right)+B_{1} .
$$

On the other hand,

$$
\pi_{B}^{-1}\left(\operatorname{ker} \psi_{3}\right)=\operatorname{ker} \psi_{3} \circ \pi_{B}=\operatorname{ker} \pi_{C} \circ \psi_{2}=\left(\psi_{2}\right)^{-1}\left(C_{1}\right) .
$$

Thus, the bottom row is exact at $B_{3}$ if and only if (2-2) holds.

Remark 2.2. In this lemma, we were interested in characterizing exactness of the bottom (interesting) row of the diagram. Lemma 3.5 of [Baum et al. 2016] does this in terms of subsets of the spectrum $\hat{B}_{2}$, which could just as well be done with subsets of Prim $B_{2}$, but we instead did it directly in terms of ideals of $B_{2}$. Note that, although the $\iota_{\bullet}$ were inclusion maps of ideals and the $\pi_{\bullet}$ were the associated quotient maps, for technical reasons we did not make the analogous assumptions regarding the middle row.

There is a standard characterization from homological algebra, namely that the bottom row is exact if and only if the top row is - this is sometimes called the nine lemma, and is an easy consequence of the snake lemma. However, this doesn't seem to lead to a simplification of the proof. 


\section{The categories and functors}

We want to study coaction functors. Among other things, we want to apply the theory we've developed in [KLQ 2013; 2016] concerning large ideals $E$ of $B(G)$. On the other hand, it is important to us in this paper for our theory to be consistent with the crossed-product functors of [Baum et al. 2016]. In particular, we want to be able to apply our coaction functors to short exact sequences.

But now a subtlety arises: some of us working in noncommutative duality for $C^{*}$-dynamical systems have grown accustomed to doing everything in the "nondegenerate" categories, where the morphisms are nondegenerate homomorphisms into multiplier algebras (possibly preserving some extra structure). But the maps in a short exact sequence

$$
0 \longrightarrow I \stackrel{\phi}{\longrightarrow} A \stackrel{\psi}{\longrightarrow} B \longrightarrow 0
$$

are not of this type, most importantly $\phi$. So, we must replace the nondegenerate category by something else. We can't just allow arbitrary homomorphisms into multiplier algebras, because they wouldn't be composable. We can't require "extendible homomorphisms" into multiplier algebras, because the inclusion of an ideal won't typically have that property. Thus, it seems we need to use the "classical category" of homomorphisms between the $C^{*}$-algebras, not into multiplier algebras. This is what [Baum et al. 2016] uses, so presumably our best chance of seamlessly connecting with their work is to do likewise.

Since most of the existing categorical theory of coactions uses nondegenerate categories, it behooves us to establish the basic theory we need in the context of the classical categories, which we do below.

One drawback to this is that the covariant homomorphisms and crossed products can't be constructed using morphisms from the classical $C^{*}$-category - so, it seems we have to abandon some of the appealing features of the nondegenerate category.

Definition 3.1. A morphism $\phi: A \rightarrow B$ in the classical category $\mathbf{C}^{*}$ of $C^{*}$-algebras is a $*$-homomorphism from $A$ to $B$ in the usual sense (no multipliers).

Definition 3.2. A morphism $\phi:(A, \delta) \rightarrow(B, \varepsilon)$ in the classical category Coact of coactions is a morphism $\phi: A \rightarrow B$ in $\mathbf{C}^{*}$ such that the diagram

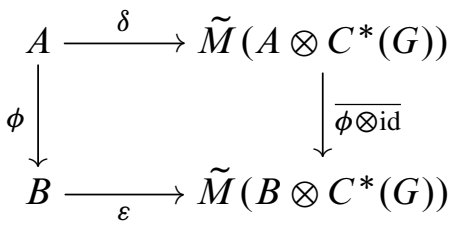

commutes, and we call $\phi$ a $\delta-\varepsilon$ equivariant homomorphism. 
To make sense of the above commuting diagram, recall that for any $C^{*}$-algebra $C$,

$$
\tilde{M}(A \otimes C)=\{m \in M(A \otimes C): m(1 \otimes C) \cup(1 \otimes C) m \subset A \otimes C\},
$$

and that for any homomorphism $\phi: A \rightarrow B$ there is a canonical extension to a homomorphism

$$
\overline{\phi \otimes \mathrm{id}}: \tilde{M}(A \otimes C) \rightarrow \tilde{M}(B \otimes C),
$$

by [Echterhoff et al. 2006, Proposition A.6]. It is completely routine to verify that C $^{*}$ and Coact are categories, i.e., there are identity morphisms and there is an associative composition.

Remark 3.3. Thus, a coaction is not itself a morphism in the classical category; this will cause no trouble.

To work in the classical category of coactions, we need to be just a little bit careful with covariant homomorphisms and crossed products. We write $w_{G}$ for the unitary element of $M\left(C_{0}(G) \otimes C^{*}(G)\right)=C_{b}\left(G, M^{\beta}\left(C^{*}(G)\right)\right)$ defined by $w_{G}(s)=s$, where we have identified $G$ with its canonical image in $M\left(C^{*}(G)\right)$, and where the superscript $\beta$ means that we use the strict topology on $M\left(C^{*}(G)\right)$.

Definition 3.4. A degenerate covariant homomorphism of a coaction $(A, \delta)$ to a $C^{*}$-algebra $B$ is a pair $(\pi, \mu)$, where $\pi: A \rightarrow M(B)$ and $\mu: C_{0}(G) \rightarrow M(B)$ are homomorphisms such that $\mu$ is nondegenerate and the diagram

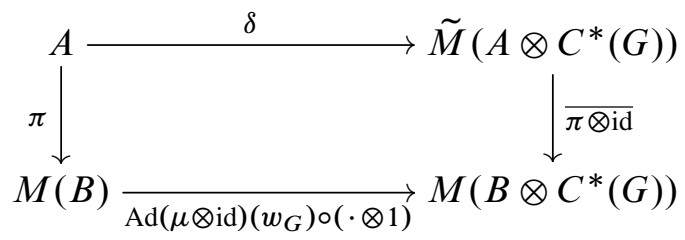

commutes, where the bottom arrow is the map $b \mapsto \operatorname{Ad}(\mu \otimes \mathrm{id})\left(w_{G}\right)(b \otimes 1)$. If $\pi: A \rightarrow M(B)$ happens to be nondegenerate, we sometimes refer to $(\pi, \mu)$ as a nondegenerate covariant homomorphism for clarity.

Remark 3.5. The homomorphisms $\pi$ and $\mu$ are not morphisms in the classical category $\mathbf{C}^{*}$; this will cause no trouble, but does present a danger of confusion.

Remark 3.6. Thus, in our new definition of degenerate covariant homomorphism, we include all the usual nondegenerate covariant homomorphisms, and we add more, allowing the homomorphism $\pi$ of $A$ (but not the homomorphism $\mu$ of $C_{0}(G)$ ) to be degenerate.

Remark 3.7. We wrote $M\left(B \otimes C^{*}(G)\right)$, rather than the relative multiplier algebra $\tilde{M}\left(B \otimes C^{*}(G)\right)$, in the above diagram, because $\overline{\pi \otimes \text { id }}$ will in general not map $\tilde{M}\left(A \otimes C^{*}(G)\right)$ into $\tilde{M}\left(B \otimes C^{*}(G)\right)$ since $\pi$ does not map $A$ into $B$. 
Although we have apparently enlarged the supply of covariant homomorphisms, in some sense we have not. In Lemma 3.8 below we use the following terminology: given $C^{*}$-algebras $A \subset B$, the idealizer of $A$ in $B$ is $\{b \in B: b A \cup A b \subset A\}$.

Lemma 3.8. Let $(\pi, \mu)$ be a degenerate covariant homomorphism of $(A, \delta)$ to $B$, as in Definition 3.4. Put

$$
B_{0}=\overline{\operatorname{span}}\left\{\pi(A) \mu\left(C_{0}(G)\right)\right\} .
$$

Then:

(1) $B_{0}=\overline{\operatorname{span}}\left\{\mu\left(C_{0}(G)\right) \pi(A)\right\}$.

(2) $B_{0}$ is a $C^{*}$-subalgebra of $M(B)$.

(3) $\pi$ and $\mu$ map into the idealizer $D$ of $B_{0}$ in $M(B)$. Let $\rho: D \rightarrow M\left(B_{0}\right)$ be the homomorphism given by

$$
\rho(m) b_{0}=m b_{0} \quad \text { for } m \in D \subset M(B), b_{0} \in B_{0} \subset B,
$$

and let $\pi_{0}=\rho \circ \pi: A \rightarrow M\left(B_{0}\right)$ and $\mu_{0}=\rho \circ \mu: C_{0}(G) \rightarrow M\left(B_{0}\right)$. Then $\left(\pi_{0}, \mu_{0}\right)$ is a nondegenerate covariant homomorphism of $(A, \delta)$ to $B_{0}$.

(4) For all $a \in A$ and $f \in C_{0}(G)$ we have

$$
\pi_{0}(a) \mu_{0}(f)=\pi(a) \mu(f) \in B_{0} .
$$

Proof. For (1), by symmetry it suffices to show that for $a \in A$ and $f \in C_{0}(G)$ we have

$$
\mu(f) \pi(a) \in B_{0},
$$

and we use an old trick from [Landstad et al. 1987, proof of Lemma 2.5]: since $A(G)$ is dense in $C_{0}(G)$, it suffices to take $f \in A(G)$, and then since $A(G)$ is a nondegenerate $C^{*}(G)$-module via $\langle y, g \cdot x\rangle=\langle x y, g\rangle$ for $x, y \in C^{*}(G), g \in A(G)$, by Cohen's factorization theorem we can write $f=g \cdot x$. Then the following approximation suffices:

$$
\begin{aligned}
& \mu(f) \pi(a)=\left\langle(\mu \otimes \mathrm{id})\left(w_{G}\right), \mathrm{id} \otimes f\right\rangle \pi(a) \\
& =\left\langle(\mu \otimes \mathrm{id})\left(w_{G}\right)(\pi(a) \otimes 1), \mathrm{id} \otimes f\right\rangle \\
& =\left\langle\overline{\pi \otimes \mathrm{id}}(\delta(a))(\mu \otimes \mathrm{id})\left(w_{G}\right), \mathrm{id} \otimes g \cdot x\right\rangle \\
& =\left\langle(\pi \otimes \mathrm{id})((1 \otimes x) \delta(a))(\mu \otimes \mathrm{id})\left(w_{G}\right), \mathrm{id} \otimes g\right\rangle \\
& \approx \sum_{i}\left\langle(\pi \otimes \mathrm{id})\left(a_{i} \otimes x_{i}\right)(\mu \otimes \mathrm{id})\left(w_{G}\right), \mathrm{id} \otimes g\right\rangle \\
& \text { for finitely many } a_{i} \in A, x_{i} \in C^{*}(G) \\
& =\sum_{i}\left\langle\left(\pi\left(a_{i}\right) \otimes x_{i}\right)(\mu \otimes \mathrm{id})\left(w_{G}\right), \mathrm{id} \otimes g\right\rangle
\end{aligned}
$$




$$
\begin{aligned}
& =\sum_{i} \pi\left(a_{i}\right)\left\langle(\mu \otimes \mathrm{id})\left(w_{G}\right), \mathrm{id} \otimes g \cdot x_{i}\right\rangle \\
& =\sum_{i} \pi\left(a_{i}\right) \mu\left(g \cdot x_{i}\right) .
\end{aligned}
$$

From (1) it follows that $B_{0}$ is a $*$-subalgebra of $B$, giving (2).

(3) It is now clear that

$$
\pi(A) B_{0} \cup B_{0} \pi(A) \subset B_{0},
$$

and similarly for $\mu$, so both $\pi$ and $\mu$ map into $D$. It is also clear that $\pi_{0}$ and $\mu_{0}$ map nondegenerately into $M\left(B_{0}\right)$. The covariance property for $\left(\pi_{0}, \mu_{0}\right)$ follows quickly from that of $(\pi, \mu)$ : if $a \in A$ then

$$
\begin{aligned}
\operatorname{Ad}\left(\mu_{0} \otimes \mathrm{id}\right)\left(w_{G}\right)\left(\pi_{0}(a) \otimes 1\right) & =(\rho \otimes \mathrm{id}) \circ \operatorname{Ad}(\mu \otimes \mathrm{id})\left(w_{G}\right)(\pi(a) \otimes 1) \\
& =(\rho \otimes \mathrm{id}) \circ \overline{\pi \otimes \mathrm{id}} \circ \delta(a) \\
& =\overline{\pi_{0} \otimes \mathrm{id}} \circ \delta(a) .
\end{aligned}
$$

(4) This follows from the construction.

Let $\left(A \rtimes_{\delta} G, j_{A}, j_{G}\right)$ be the usual crossed product of the coaction $(A, \delta)$, i.e., $\left(j_{A}, j_{G}\right)$ is a nondegenerate covariant homomorphism of $(A, \delta)$ to $A \rtimes_{\delta} G$ that is universal in the sense that if $(\pi, \mu)$ is any nondegenerate covariant homomorphism of $(A, \delta)$ to a $C^{*}$-algebra $B$, then there is a unique homomorphism $\pi \times \mu: A \rtimes_{\delta} G \rightarrow M(B)$ such that

$$
\begin{aligned}
& \pi \times \mu \circ j_{A}=\pi, \\
& \pi \times \mu \circ j_{G}=\mu,
\end{aligned}
$$

equivalently such that

$$
\pi \times \mu\left(j_{A}(a) j_{G}(f)\right)=\pi(a) \mu(f) \text { for all } a \in A, f \in C_{0}(G) .
$$

Corollary 3.9. With the above notation, $\left(j_{A}, j_{G}\right)$ is also universal among degenerate covariant homomorphisms (in the sense of Definition 3.4). More precisely: for any degenerate covariant homomorphism $(\pi, \mu)$ of $(A, \delta)$ to $B$ as in Definition 3.4, there is a unique homomorphism $\pi \times \mu: A \rtimes_{\delta} G \rightarrow M(B)$ satisfying (3-1).

Proof. Let $\pi_{0}, \mu_{0}, B_{0}$ be as in the preceding lemma. Then we have a unique homomorphism $\pi_{0} \times \mu_{0}: A \rtimes_{\delta} G \rightarrow M\left(B_{0}\right)$ such that

$$
\pi_{0} \times \mu_{0}\left(j_{A}(a) j_{G}(f)\right)=\pi_{0}(a) \mu_{0}(f) \text { for all } a \in A, f \in C_{0}(G) .
$$

By construction we have $\pi \times \mu\left(A \rtimes_{\delta} G\right) \subset B_{0}$. Since $B_{0} \subset M(B)$, we can regard $\pi_{0}$ as a homomorphism $\pi: A \rightarrow M(B)$, and similarly for $\mu: C_{0}(G) \rightarrow M(B)$. Then 
we regard $\pi_{0} \times \mu_{0}$ as a homomorphism $\pi \times \mu: A \rtimes_{\delta} G \rightarrow M(B)$, and trivially (3-1) holds. Since $\pi_{0}(a) \mu_{0}(f)=\pi(a) \mu(f) \in B_{0}$ for all $a \in A, f \in C_{0}(G)$, the homomorphism $\pi \times \mu$ is unique.

Similarly, and more easily, for actions:

Definition 3.10. A morphism $\phi:(A, \alpha) \rightarrow(B, \beta)$ in the classical category Act of actions is a morphism $\phi: A \rightarrow B$ in $\mathbf{C}^{*}$ such that

$$
\beta_{s} \circ \phi=\phi \circ \alpha_{s} \quad \text { for all } s \in G .
$$

Definition 3.11. A degenerate covariant homomorphism of an action $(A, \alpha)$ to a $C^{*}$-algebra is a pair $(\pi, u)$, where $\pi: A \rightarrow M(B)$ is a homomorphism and $u: G \rightarrow M(B)$ is a strictly continuous unitary homomorphism such that

$$
\pi \circ \alpha_{s}=\operatorname{Ad} u_{s} \circ \pi \quad \text { for all } s \in G .
$$

We call $(\pi, u)$ nondegenerate if $\pi: A \rightarrow M(B)$ is.

Lemma 3.12. Let $(\pi, u)$ be a degenerate covariant homomorphism of an action $(A, \alpha)$ to $B$, and put

$$
B_{0}=\overline{\operatorname{span}}\left\{\pi(A) u\left(C^{*}(G)\right)\right\},
$$

where we use the same notation $u$ for the associated nondegenerate homomorphism $u: C^{*}(G) \rightarrow M(B)$. Then:

(1) $B_{0}=\overline{\operatorname{span}}\left\{u\left(C^{*}(G)\right) \pi(A)\right\}$.

(2) $B_{0}$ is a $C^{*}$-subalgebra of $M(B)$.

(3) $\pi$ and $u$ map into the idealizer $D$ of $B_{0}$ in $M(B)$. Let $\rho: D \rightarrow M\left(B_{0}\right)$ be the homomorphism given by

$$
\rho(m) b_{0}=m b_{0} \quad \text { for } m \in D \subset M(B), b_{0} \in B_{0} \subset B,
$$

and let $\pi_{0}=\rho \circ \pi: A \rightarrow M\left(B_{0}\right)$ and $u_{0}=\rho \circ u: G \rightarrow M\left(B_{0}\right)$. Then $\left(\pi_{0}, u_{0}\right)$ is a nondegenerate covariant homomorphism of $(A, \alpha)$ to $B_{0}$.

(4) For all $a \in A$ and $c \in C^{*}(G)$ we have

$$
\pi_{0}(a) u_{0}(c)=\pi(a) u(c) \in B_{0} .
$$

Let $\left(A \rtimes_{\alpha} G, i_{A}, i_{G}\right)$ be the usual crossed product of the action $(A, \alpha)$, i.e., $\left(i_{A}, i_{G}\right)$ is a nondegenerate covariant homomorphism of $(A, \alpha)$ to $A \rtimes_{\alpha} G$ that is universal in the sense that if $(\pi, u)$ is any nondegenerate covariant homomorphism of $(A, \alpha)$ to a $C^{*}$-algebra $B$, then there is a unique homomorphism $\pi \times u: A \rtimes_{\alpha} G \rightarrow M(B)$ such that

$$
\pi \times u\left(i_{A}(a) i_{G}(c)\right)=\pi(a) u(c) \quad \text { for all } a \in A, c \in C^{*}(G) .
$$


Corollary 3.13. With the above notation, $\left(i_{A}, i_{G}\right)$ is also universal among degenerate covariant homomorphisms (in the sense of Definition 3.4): for any degenerate covariant homomorphism $(\pi, u)$ of $(A, \alpha)$ to $B$ as in Definition 3.11, there is a unique homomorphism $\pi \times u: A \rtimes_{\alpha} G \rightarrow M(B)$ satisfying (3-2).

If $\phi:(A, \delta) \rightarrow(B, \varepsilon)$ is a morphism in Coact, then a routine adaptation of the usual arguments shows that we get a morphism

$$
\phi \rtimes G=\left(j_{B} \circ \phi\right) \times j_{G}^{B}:\left(A \rtimes_{\delta} G, \widehat{\delta}\right) \rightarrow\left(B \rtimes_{\varepsilon} G, \widehat{\varepsilon}\right)
$$

in Act, and similarly if $\phi:(A, \alpha) \rightarrow(B, \beta)$ is a morphism in Act we get a morphism

$$
\phi \rtimes G=\left(i_{B} \circ \phi\right) \times i_{G}^{B}:\left(A \rtimes_{\alpha} G, \widehat{\alpha}\right) \rightarrow\left(B \rtimes_{\beta} G, \widehat{\beta}\right)
$$

in Coact. Thus we have crossed-product functors between the classical categories of coactions and actions.

It is also routine to verify that if $(A, \delta)$ is a coaction then the canonical surjection

$$
\Phi: A \rtimes_{\delta} G \rtimes_{\hat{\delta}} G \rightarrow A \otimes \mathcal{K}
$$

is a natural transformation between the double crossed-product functor and stabilization. $^{1}$

We need to check that normalization and maximalization behave appropriately in the new coaction category.

Maximalization. A maximalization of a coaction $(A, \delta)$ consists of a maximal coaction $\left(A^{m}, \delta^{m}\right)$ and a surjective morphism $q^{m}:\left(A^{m}, \delta^{m}\right) \rightarrow(A, \delta)$ in Coact such that

$$
q^{m} \rtimes G: A^{m} \rtimes_{\delta^{m}} G \rightarrow A \rtimes_{\delta} G
$$

is an isomorphism. Existence of maximalizations is established in [Fischer 2004, Theorem 6.4; Echterhoff et al. 2004, Theorem 3.3].

To make maximalization into a functor on the classical category of coactions, we note that the argument of [Fischer 2004, proof of Lemma 6.2] carries over to give an appropriate version of the universal property: given coactions $(A, \delta)$ and $(B, \varepsilon)$, with $\varepsilon$ maximal, and a morphism $\phi:(B, \varepsilon) \rightarrow(A, \delta)$ in Coact, there is a unique morphism $\widetilde{\phi}$ in Coact making the diagram

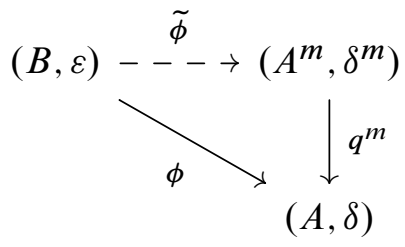

\footnotetext{
${ }^{1}$ It is completely routine to verify that stabilization $A \mapsto A \otimes \mathcal{K}$ is a functor on the classical category $\mathbf{C}^{*}$.
} 
commute. Thus, given a morphism $\phi:(A, \delta) \rightarrow(B, \varepsilon)$ in Coact, there is a unique morphism $\phi^{m}$ making the diagram

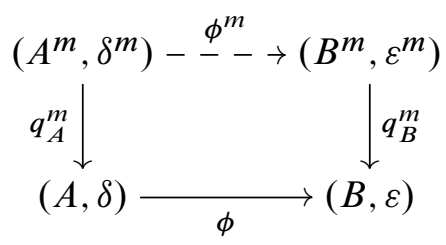

commute in Coact. Uniqueness makes the assignments $\phi \mapsto \phi^{m}$ functorial, and the maximalizing maps $q^{m}$ give a natural transformation from the maximalization functor to the identity functor. Also, the universal property implies that the maximalization functor is faithful, i.e., if $\phi, \psi:(A, \delta) \rightarrow(B, \varepsilon)$ are distinct morphisms in Coact, then the maximalizations $\phi^{m}, \psi^{m}:\left(A^{m}, \delta^{m}\right) \rightarrow\left(B^{m}, \varepsilon^{m}\right)$ are also distinct.

Remark 3.14. It is important for us that maximalization is a functor; however, when we refer to $\left(A^{m}, \delta^{m}\right)$ as "the" maximalization of a coaction $(A, \delta)$, we do not have in mind a specific $C^{*}$-algebra $A^{m}$, rather we regard the maximalization as being characterized up to isomorphism by its universal properties, but for the purpose of having a functor we imagine that a choice of maximalization has been made for every coaction - any other choices would give a naturally isomorphic functor. On the other hand, whenever we have a maximal coaction $(B, \varepsilon)$, we may call a morphism $\phi:(B, \varepsilon) \rightarrow(A, \delta)$ with the defining property a maximalization of $(A, \delta)$.

Normalization. A normalization of a coaction $(A, \delta)$ consists of a normal coaction $\left(A^{n}, \delta^{n}\right)$ and a surjective morphism $\Lambda:(A, \delta) \rightarrow\left(A^{n}, \delta^{n}\right)$ in Coact such that

$$
\Lambda \rtimes G: A \rtimes_{\delta} G \rightarrow A^{n} \rtimes_{\delta^{n}} G
$$

is an isomorphism. Existence of normalizations is established in [Quigg 1994, Proposition 2.6].

To make normalization into a functor on the classical category of coactions, we note that [Echterhoff et al. 2004, Lemma 2.1] says that, given a morphism $\phi:(A, \delta) \rightarrow(B, \varepsilon)$ in Coact, there is a unique morphism $\phi^{n}$ making the diagram

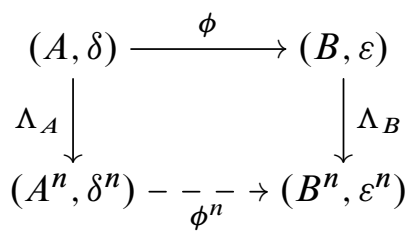

commute in Coact. Uniqueness makes the assignments $\phi \mapsto \phi^{n}$ functorial, and the normalizing maps $\Lambda$ give a natural transformation from the identity functor to the normalization functor. 
Remark 3.15. The comments of Remark 3.14 can be adapted in an obvious way to normalization, and also to crossed products, etc. There are numerous "natural" relationships among such functors; for example, maximalization is naturally isomorphic to the composition

$$
(A, \delta) \mapsto\left(A^{n}, \delta\right) \mapsto\left(A^{n m}, \delta^{n m}\right)
$$

of normalization followed by maximalization, and the dual coaction $\hat{\alpha}^{n}$ on the reduced crossed product $A \rtimes_{\alpha, r} G$ of an action $(A, \alpha)$ is naturally isomorphic to the normalization of the dual coaction $\hat{\alpha}$ on the full crossed product $A \rtimes_{\alpha} G$ [Echterhoff et al. 2006, Proposition A.61].

The normalization $\Lambda:(A, \delta) \rightarrow\left(A^{n}, \delta^{n}\right)$ of a maximal coaction is also a maximalization of the normal coaction $\delta^{n}$. It follows that the normalization functor is faithful, i.e., if $\phi, \psi:(A, \delta) \rightarrow(B, \varepsilon)$ are distinct morphisms in Coact, then the normalizations $\phi^{n}, \psi^{n}:\left(A^{n}, \delta^{n}\right) \rightarrow\left(B^{n}, \varepsilon^{n}\right)$ are also distinct. It follows from this and surjectivity of the normalizing maps $\Lambda_{A}:(A, \delta) \rightarrow\left(A^{n}, \delta^{n}\right)$ that the normalizing maps are monomorphisms in the category Coact, i.e., if $\phi, \psi:(A, \delta) \rightarrow(B, \varepsilon)$ are distinct morphisms in Coact, then the compositions $\Lambda_{B} \circ \phi, \Lambda_{B} \circ \psi:(A, \delta) \rightarrow\left(B^{n}, \varepsilon^{n}\right)$ are also distinct. ${ }^{2}$

Exact sequences. It is crucial for us to note that in each of the classical categories $\mathbf{C}^{*}$, Coact, and Act there is an obvious concept of short exact sequence. Nilsen [1999] develops the basic theory of short exact sequences for coactions and crossed products. We briefly outline the essential facts here.

Definition 3.16. Let $(A, \delta)$ be a coaction. An ideal $I$ of $A$ is strongly $\delta$-invariant if

$$
\overline{\operatorname{span}}\left\{\delta(I)\left(1 \otimes C^{*}(G)\right)\right\}=I \otimes C^{*}(G) .
$$

We will normally just write invariant to mean strongly invariant.

Nilsen proves [1999, Propositions 2.1 and 2.2, Theorem 2.3] (see also [Landstad et al. 1987, Proposition 4.8]) that, using her conventions, if $I$ is strongly invariant then:

(1) $\delta$ restricts to a coaction $\delta_{I}$ on $I$.

(2) $I \rtimes_{\delta_{I}} G$ is (canonically isomorphic to) an ideal of $A \rtimes_{\delta} G$.

(3) $I$ is weakly $\delta$-invariant, i.e., $\delta$ descends to a coaction $\delta^{I}$ on $A / I$.

(4) $0 \rightarrow I \rtimes_{\delta_{I}} G \rightarrow A \rtimes_{\delta} G \rightarrow(A / I) \rtimes_{\delta^{I}} G \rightarrow 0$ is a short exact sequence in the classical category $\mathbf{C}^{*}$.

${ }^{2}$ The analogous fact for the nondegenerate category of coactions is [Bédos et al. 2011, Corollary 6.1.20]. 
We point out that Nilsen had to do a bit of work to map $I \rtimes_{\delta_{I}} G$ into $A \rtimes_{\delta} G$; in our framework with the classical categories, we just note that the inclusion $\phi: I \hookrightarrow A$ is $\delta_{I}-\delta$ equivariant, hence gives a morphism in Coact, so we can apply the functor $\mathrm{CP}$ to get a morphism

$$
\phi \rtimes G: I \rtimes_{\delta_{I}} G \rightarrow A \rtimes_{\delta} G \quad \text { in } \mathbf{C}^{*} .
$$

Definition 3.17. A functor between any two of the categories $\mathbf{C}^{*}$, Coact, Act is exact if it preserves short exact sequences.

Example 3.18. The full crossed-product functor

$$
\begin{aligned}
(A, \alpha) & \mapsto\left(A \rtimes_{\alpha} G, \widehat{\alpha}\right), \\
\phi & \mapsto \phi \rtimes G
\end{aligned}
$$

from Act to Coact is exact [Green 1978, Proposition 12]. However, the reduced crossed-product functor is not exact, due to Gromov's examples of nonexact groups.

Example 3.19. The crossed-product functor

$$
\begin{aligned}
(A, \delta) & \mapsto\left(A \rtimes_{\delta} G, \widehat{\delta}\right), \\
\phi & \mapsto \phi \rtimes G
\end{aligned}
$$

from Coact to Act is exact [Nilsen 1999, Theorem 2.3].

Example 3.20. The stabilization functor

$$
\begin{aligned}
& A \mapsto A \otimes \mathcal{K}, \\
& \phi \mapsto \phi \otimes \mathrm{id}
\end{aligned}
$$

on $\mathbf{C}^{*}$ is exact.

\section{Coaction functors}

Baum, Guentner and Willett [Baum et al. 2016] defined a crossed-product as a functor $(B, \alpha) \mapsto B \rtimes_{\alpha, \tau} G$, from the category of actions to the category of $C^{*}$ algebras, equipped with natural transformations

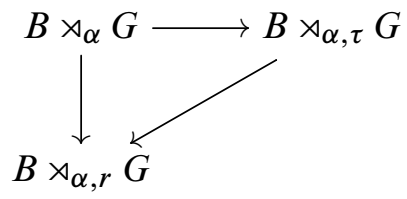

where the vertical arrow is the regular representation, such that the horizontal arrow is surjective. 
Our predilection is to decompose such a crossed-product functor as a composition

$$
(B, \alpha) \mapsto\left(B \rtimes_{\alpha} G, \hat{\alpha}\right) \mapsto B \rtimes_{\alpha, \tau} G,
$$

where the first arrow is the full crossed product and the second arrow depends only upon the dual coaction $\widehat{\alpha}$. Our approach will require the target $C^{*}$-algebra $B \rtimes_{\alpha, \tau} G$ to carry a quotient of the dual coaction. Thus, it is certainly not obvious that our techniques can handle all crossed-product functors of [Baum et al. 2016], because that paper does not require the crossed products $B \rtimes_{\alpha, \tau} G$ to have coactions, and even if they all do, there is no reason to believe that the crossed-product functor factors in this way. Nevertheless, we think that it is useful to study crossed-product functors that do factor, and thus we can focus upon the second functor, where all the action stays within the realm of coactions. The following definition is adapted more or less directly from [loc. cit., Definition 2.1]:

Definition 4.1. A coaction functor is a functor $\tau:(A, \delta) \mapsto\left(A^{\tau}, \delta^{\tau}\right)$ on the category of coactions, together with a natural transformation $q^{\tau}$ from maximalization to $\tau$ such that for every coaction $(A, \delta)$,

(1) $q_{A}^{\tau}: A^{m} \rightarrow A^{\tau}$ is surjective, and

(2) $\operatorname{ker} q_{A}^{\tau} \subset \operatorname{ker} \Lambda_{A^{m}}$.

Example 4.2. (1) Maximalization $(A, \delta) \mapsto\left(A^{m}, \delta^{m}\right)$ is a coaction functor, with natural surjections given by the identity maps $\operatorname{id}_{A^{m}}$.

(2) Normalization $(A, \delta) \mapsto\left(A^{n}, \delta^{n}\right)$ is a coaction functor, with natural surjections $\Lambda_{A^{m}}: A^{m} \rightarrow A^{n}$.

(3) The identity functor is a coaction functor, with natural surjections $q_{A}^{m}: A^{m} \rightarrow A$.

Lemma 4.3. If $\tau$ is a coaction functor, then for every coaction $(A, \delta)$ there is a unique $\delta^{\tau}-\delta^{n}$ equivariant surjection $\Lambda_{A}^{\tau}$ making the diagram

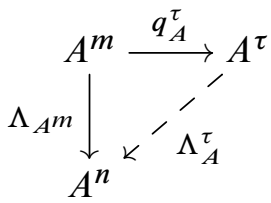

commute. Moreover, $\Lambda^{\tau}$ is a natural transformation from $\tau$ to normalization.

Proof. The first statement follows immediately from the definitions. To verify that $\Lambda^{\tau}$ is a natural transformation, we must show that the homomorphisms $\Lambda^{\tau}$

(1) are morphisms of coactions, and

(2) are natural. 
(1) In the commuting triangle (4-1), we must show that $\Lambda_{A}^{\tau}$ is a $B(G)$-module map, but this follows since $\Lambda_{A}$ and $q_{A}^{\tau}$ are module maps and $q_{A}^{\tau}$ is surjective.

(2) For the naturality, let $\phi:(A, \delta) \rightarrow(B, \varepsilon)$ be a morphism in the category of coactions. Consider the diagram

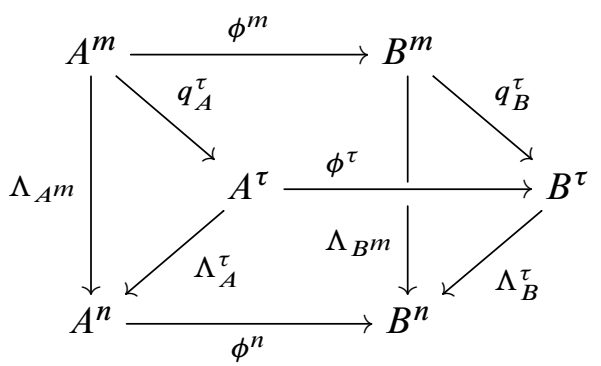

We need to know that the lower quadrilateral, with horizontal and southwest arrows, commutes, and this follows from surjectivity of $q_{A}^{\tau}$ and commutativity of the other two quadrilaterals and the two triangles.

Corollary 4.4. If $\tau$ is a coaction functor, then in (4-1) we have

(1) $q^{\tau}: A^{m} \rightarrow A^{\tau}$ is a maximalization of $\delta^{\tau}$, and

(2) $\Lambda^{\tau}: A^{\tau} \rightarrow A^{n}$ is a normalization of $\delta^{\tau}$.

Proof. Taking crossed products in (4-1), we get a commutative diagram

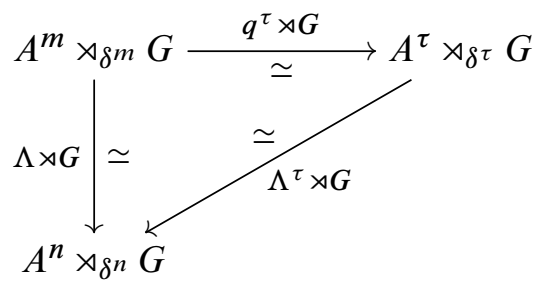

where the horizontal arrow is surjective because $q^{\tau}$ is, and is injective because of the vertical isomorphism, and then the diagonal arrow is an isomorphism because the other two arrows are. Thus $q^{\tau}$ and $\Lambda^{\tau}$ satisfy the defining properties of maximalization and normalization, respectively.

Remark 4.5. Caution: it might seem that $\tau$ should factor through the maximalization functor, at least up to natural isomorphism. This would entail, in particular, that

$$
\left(A^{m \tau}, \delta^{m \tau}\right) \cong\left(A^{\tau}, \delta^{\tau}\right) \text { for every coaction }(A, \delta) .
$$

But this is violated with $\tau=\mathrm{id}$. 
Notation 4.6. With the above notation, we define an ideal of $A^{m}$ by

$$
A_{\tau}^{m}:=\operatorname{ker} q_{A}^{\tau} .
$$

Note that for the maximalization functor $m$ we have $A_{m}^{m}=\{0\}$, while for the normalization functor $n$ the associated ideal $A_{n}^{m}$ is the kernel of the normalization $\operatorname{map} \Lambda_{A^{m}}: A^{m} \rightarrow A^{m n} \cong A^{n}$.

Partial ordering of coaction functors. Baum, Guentner and Willett [Baum et al. 2016, p. 8] define one crossed-product functor $\sigma$ to be smaller than another one $\tau$ if the natural surjection $A \rtimes_{\alpha, \tau} G \rightarrow A \rtimes_{\alpha, r} G$ factors through the $\sigma$-crossed product.

We adapt this definition of partial order to coaction functors, but "from the top rather than toward the bottom".

Definition 4.7. If $\sigma$ and $\tau$ are coaction functors, then $\sigma$ is smaller than $\tau$, written $\sigma \leq \tau$, if for every coaction $(A, \delta)$ we have

$$
A_{\tau}^{m} \subset A_{\sigma}^{m} .
$$

Lemma 4.8. For coaction functors $\sigma, \tau$, the following are equivalent:

(1) $\sigma \leq \tau$.

(2) For every coaction $(A, \delta)$ there is a homomorphism $\Gamma^{\tau, \sigma}$ making the diagram

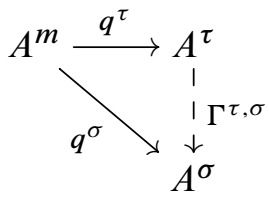

commute.

(3) For every coaction $(A, \delta)$ there is a homomorphism $\Gamma^{\tau, \sigma}$ making the diagram

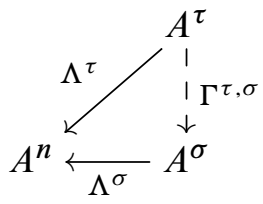

commute.

Moreover, if these equivalent conditions hold then $\Gamma^{\tau, \sigma}$ is unique, is surjective, and is a natural transformation from $\tau$ to $\sigma$.

Proof. (1) is equivalent to (2) since $A_{\tau}^{m}=\operatorname{ker} q^{\tau}$ and $A_{\sigma}^{m}=\operatorname{ker} q^{\sigma}$. Moreover, (1) implies that $\Gamma^{\tau, \sigma}$ is unique and is surjective, since the maps $q^{\tau}$ are surjective. 
Assume (3). Consider the combined diagram

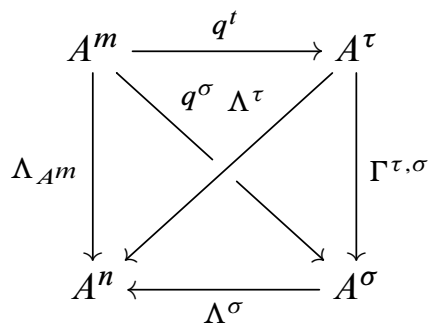

The upper left and lower left triangles commute by definition of coaction functor, and the lower right triangle commutes by assumption. Thus the upper right triangle commutes after postcomposing with $\Lambda^{\sigma}$. Since the latter map is a normalizer, by [Bédos et al. 2011, Corollary 6.1.20] it is a monomorphism in the category of coactions. Thus the upper right triangle commutes.

Similarly (but more easily), assuming (2), the lower right triangle in the diagram (4-2) commutes because it commutes after precomposing with the surjection $q^{\tau}$.

Naturality of $\Gamma^{\tau, \sigma}$ is proved by virtually the same argument as in Lemma 4.3.

The following is a coaction-functor analogue of [Baum et al. 2016, Lemma 3.7], and we adapt their argument:

Theorem 4.9. Every nonempty collection $\mathcal{T}$ of coaction functors has a greatest lower bound $\sigma$ with respect to the above partial ordering, characterized by

$$
A_{\sigma}^{m}=\underset{\tau \in \mathcal{T}}{\operatorname{span}_{\tau}} A_{\tau}^{m}
$$

for every coaction $(A, \delta)$.

Proof. Let $(A, \delta)$ be a coaction, Then the ideal

$$
A_{\sigma}^{m}:=\overline{\operatorname{span}}_{\tau \in \mathcal{T}} A_{\tau}^{m}
$$

of $A^{m}$ is contained in the kernel of the normalization map $\Lambda_{A^{m}}$. Put

$$
A^{\sigma}=A^{m} / A_{\sigma}^{m},
$$

and let

$$
q_{A}^{\sigma}: A^{m} \rightarrow A^{\sigma}
$$

be the quotient map.

$A_{\tau}^{m}$ is a weakly $\delta^{m}$-invariant ideal of $A^{m}$ for all $\tau \in \mathcal{T}$, so for all $f \in B(G)$ we have

$$
f \cdot A_{\tau}^{m} \subset A_{\tau}^{m} \subset A_{\sigma}^{m},
$$

and it follows that $f \cdot A_{\sigma}^{m} \subset A_{\sigma}^{m}$, i.e., $A_{\sigma}^{m}$ is a weakly $\delta^{m}$-invariant ideal. Thus $q^{\sigma}$ is equivariant for $\delta^{m}$ and a unique coaction $\delta^{\sigma}$ on $A^{\sigma}$. 
We now have assignments

$$
(A, \delta) \mapsto\left(A^{\sigma}, \delta^{\sigma}\right)
$$

on objects, and we need to handle morphisms. Thus, let $\phi:(A, \delta) \rightarrow(B, \varepsilon)$ be a morphism of coactions; i.e., $\phi: A \rightarrow B$ is a $\delta-\varepsilon$ equivariant homomorphism. Since

$$
A_{\tau}^{m} \subset\left(\phi^{m}\right)^{-1}\left(B_{\tau}^{m}\right) \subset\left(\phi^{m}\right)^{-1}\left(B_{\sigma}^{m}\right) \text { for all } \tau \in \mathcal{T},
$$

we have

$$
\operatorname{ker} q_{A}^{\sigma}=A_{\sigma}^{m}=\overline{\operatorname{span}}_{\tau \in \mathcal{T}} A_{\tau}^{m} \subset\left(\phi^{m}\right)^{-1}\left(B_{\sigma}^{m}\right)=\operatorname{ker} q_{B}^{\sigma} \circ \phi^{m} .
$$

Thus there is a unique homomorphism $\phi^{\sigma}$ making the diagram

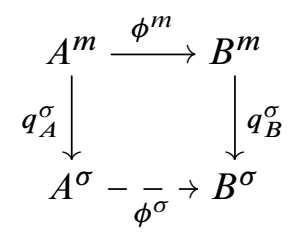

commute. Moreover, $\phi^{\sigma}$ is $\delta^{\sigma}-\varepsilon^{\sigma}$ equivariant because the other three maps are and $q_{A}^{\sigma}$ is surjective.

We need to verify that the assignments $\phi \mapsto \phi^{\sigma}$ of morphisms are functorial. Obviously identity morphisms are preserved. For compositions, let

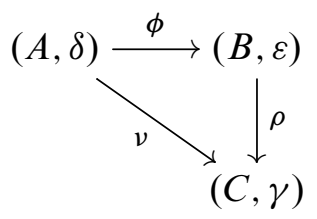

be a commuting diagram of coactions. Consider the diagram

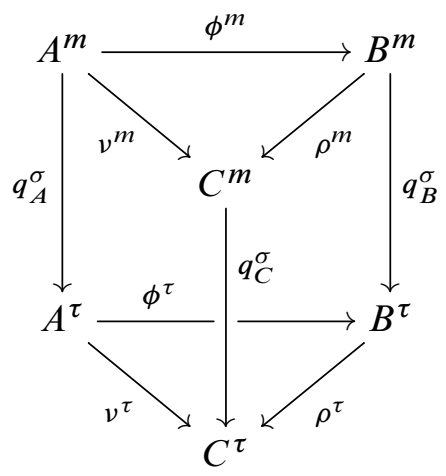


The three vertical quadrilaterals and the top triangle commute, and $q_{A}^{\sigma}$ is surjective. It follows that the bottom triangle commutes, and we have shown that composition is preserved.

Thus we have a functor $\sigma$ on the category of coactions. Moreover, $\sigma$ is a coaction functor, since the surjections $q^{\sigma}$ have small kernels and the commuting diagram (4-3) shows that $q^{\sigma}$ gives a natural transformation from maximalization to $\sigma$. By construction, $\sigma$ is a greatest lower bound for $\mathcal{T}$.

Exact coaction functors. As a special case of our general Definition 3.17, we explicitly record:

Definition 4.10. A coaction functor $\tau$ is exact if for every short exact sequence

$$
0 \longrightarrow(I, \gamma) \stackrel{\phi}{\longrightarrow}(A, \delta) \stackrel{\psi}{\longrightarrow}(B, \varepsilon) \longrightarrow 0
$$

of coactions the associated sequence

$$
0 \longrightarrow\left(I^{\tau}, \gamma^{\tau}\right) \stackrel{\phi^{\tau}}{\longrightarrow}\left(A^{\tau}, \delta^{\tau}\right) \stackrel{\psi^{\tau}}{\longrightarrow}\left(B^{\tau}, \varepsilon^{\tau}\right) \longrightarrow 0
$$

is exact.

Theorem 4.11. The maximalization functor is exact.

Proof. Let

$$
0 \longrightarrow(I, \gamma) \stackrel{\phi}{\longrightarrow}(A, \delta) \stackrel{\psi}{\longrightarrow}(B, \varepsilon) \longrightarrow 0
$$

be an exact sequence of coactions. Taking crossed products twice, we get an exact sequence

$$
0 \longrightarrow I \rtimes_{\gamma} G \rtimes_{\widehat{\gamma}} G \stackrel{\phi \rtimes G \rtimes G}{\longrightarrow} A \rtimes_{\delta} G \rtimes_{\widehat{\delta}} G \stackrel{\psi \rtimes G \rtimes G}{\longrightarrow} B \rtimes_{\varepsilon} G \rtimes_{\widehat{\varepsilon}} G \longrightarrow 0 .
$$

Since the identity functor on coactions is a coaction functor, we get an isomorphic sequence

$$
\begin{aligned}
0 \longrightarrow I^{m} \rtimes_{\gamma^{m}} G \rtimes_{\gamma^{m}} G \stackrel{\phi^{m} \rtimes G \rtimes G}{\longrightarrow} A^{m} \rtimes_{\delta^{m}} G \rtimes_{\delta^{m}} G \\
\stackrel{\psi^{m} \rtimes G \rtimes G}{\longrightarrow} B^{m} \rtimes_{\varepsilon^{m}} G \rtimes_{\varepsilon^{m}} G \longrightarrow 0,
\end{aligned}
$$

which is therefore also exact. Since the canonical surjection $\Phi$ is a natural transformation from the double crossed-product functor to the stabilization functor, and since the coactions are now maximal, we get an isomorphic sequence

$$
0 \longrightarrow I^{m} \otimes \mathcal{K} \stackrel{\phi^{m} \otimes \mathrm{id}}{\longrightarrow} A^{m} \otimes \mathcal{K} \stackrel{\psi^{m} \otimes \mathrm{id}}{\longrightarrow} B^{m} \otimes \mathcal{K} \longrightarrow 0,
$$

which is therefore also exact. Since $\mathcal{K}$ is an exact $C^{*}$-algebra,

$$
\left(\operatorname{ker} \phi^{m}\right) \otimes \mathcal{K}=\operatorname{ker}\left(\phi^{m} \otimes \mathrm{id}\right)=\{0\},
$$


so $\operatorname{ker} \phi^{m}=\{0\}$, and similarly

$\left(\operatorname{ker} \psi^{m}\right) \otimes \mathcal{K}=\operatorname{ker}\left(\psi^{m} \otimes \mathrm{id}\right)=\left(\phi^{m} \otimes \mathrm{id}\right)\left(I^{m} \otimes \mathcal{K}\right)=\phi^{m}\left(I^{m}\right) \otimes \mathcal{K}$,

so, because $\phi^{m}\left(I^{m}\right) \subset \operatorname{ker} \psi^{m}$ by functoriality, we must have $\phi^{m}\left(I^{m}\right)=\operatorname{ker} \psi^{m}$. Therefore the sequence

$$
0 \longrightarrow I^{m} \stackrel{\phi^{m}}{\longrightarrow} A^{m} \stackrel{\psi^{m}}{\longrightarrow} B^{m} \longrightarrow 0
$$

is exact.

Theorem 4.12. A coaction functor $\tau$ is exact if and only if for any short exact sequence

$$
0 \longrightarrow\left(I, \delta_{I}\right) \stackrel{\phi}{\longrightarrow}(A, \delta) \stackrel{\psi}{\longrightarrow}\left(B, \delta^{I}\right) \longrightarrow 0
$$

of coactions, both

$$
\phi^{m}\left(I_{\tau}^{m}\right)=\phi^{m}\left(I^{m}\right) \cap A_{\tau}^{m}
$$

and

$$
\phi^{m}\left(I^{m}\right)+A_{\tau}^{m}=\left(\psi^{m}\right)^{-1}\left(B_{\tau}^{m}\right)
$$

hold.

Proof. We have a commutative diagram

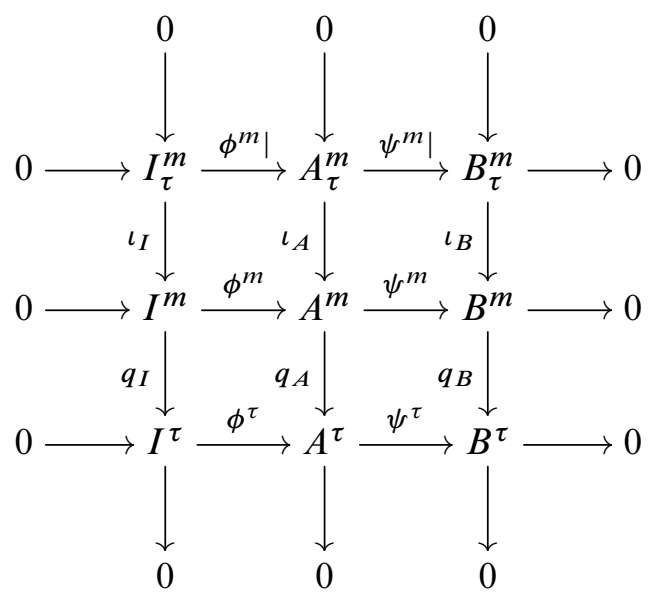

in which the columns are exact by definition, and the middle row is exact by Theorem 4.11. Thus the result follows immediately from Lemma 2.1.

Morita compatible coaction functors. If we have coactions $(A, \delta)$ and $(B, \varepsilon)$, and a $\delta-\varepsilon$ compatible coaction $\zeta$ on an $A-B$ imprimitivity bimodule $X$, we'll say that $(X, \zeta)$ is an $(A, \delta)-(B, \varepsilon)$ imprimitivity bimodule. 
Example 4.13. The double dual bimodule coaction

$$
(Y, \eta):=\left(X \rtimes_{\zeta} G \rtimes_{\hat{\zeta}} G, \widehat{\hat{\zeta}}\right)
$$

is an

$$
\left(A \rtimes_{\delta} G \rtimes_{\widehat{\delta}} G, \widehat{\hat{\delta}}\right)-\left(B \rtimes_{\varepsilon} G \rtimes_{\widehat{\varepsilon}} G, \widehat{\widehat{\varepsilon}}\right)
$$

imprimitivity bimodule. Since the identity functor on coactions is a coaction functor, $(Y, \eta)$ becomes an

$$
\left(A^{m} \rtimes_{\delta^{m}} G \rtimes_{\delta^{\hat{m}}} G, \delta^{\widehat{\hat{m}}}\right)-\left(B^{m} \rtimes_{\mathcal{E}^{m}} G \rtimes_{\mathcal{E}^{\hat{m}}} G, \varepsilon^{\widehat{\hat{m}}}\right)
$$

imprimitivity bimodule. Since maximalizations satisfy full-crossed-product duality, $(Y, \eta)$ becomes, after replacing the double dual coactions by exterior equivalent coactions, an

$$
\left(A^{m} \otimes \mathcal{K}, \delta^{m} \otimes_{*} \text { id }\right)-\left(B^{m} \otimes \mathcal{K}, \varepsilon^{m} \otimes_{*} \text { id }\right)
$$

imprimitivity bimodule (see [Echterhoff et al. 2004, Lemma 3.6]).

We need the following basic lemma, which is probably folklore, although we could not find it in the literature. Our formulation is partially inspired by Fischer's treatment of relative commutants of $\mathcal{K}$ [Fischer 2004, Section 3].

Lemma 4.14. Let $A$ and $B$ be $C^{*}$-algebras, and let $Y$ be an $(A \otimes \mathcal{K})-(B \otimes \mathcal{K})$ imprimitivity bimodule. Define

$$
X=\left\{m \in M(Y):\left(1_{A} \otimes k\right) \cdot m=m \cdot\left(1_{B} \otimes k\right) \in Y \text { for all } k \in \mathcal{K}\right\} .
$$

Then:

(1) $X$ is an $\left(A \otimes 1_{\mathcal{K}}\right)-\left(B \otimes 1_{\mathcal{K}}\right)$ submodule of $M(Y)$.

(2) $\overline{\operatorname{span}}\langle X, X\rangle_{M(B \otimes \mathcal{K})}=B \otimes 1_{\mathcal{K}}$.

(3) $\overline{\operatorname{span}}_{M(A \otimes \mathcal{K})}\langle X, X\rangle=A \otimes 1_{\mathcal{K}}$.

Thus $X$ becomes an $A-B$ imprimitivity bimodule in an obvious way, and moreover there is a unique $(A \otimes \mathcal{K})-(B \otimes \mathcal{K})$ imprimitivity bimodule isomorphism

$$
\theta: X \otimes \mathcal{K} \stackrel{\cong}{\longrightarrow} Y
$$

such that

$$
\theta(m \otimes k)=m \cdot\left(1_{B} \otimes k\right) \quad \text { for } m \in X, k \in \mathcal{K} .
$$

Lemma 4.15. Given coactions $(A, \delta)$ and $(B, \varepsilon)$, and a $\delta-\varepsilon$ compatible coaction $\zeta$ on an $A-B$ imprimitivity bimodule $X$, let $(Y, \eta)$ be the

$$
\left(A^{m} \otimes \mathcal{K}, \delta^{m} \otimes_{*} \mathrm{id}\right)-\left(B^{m} \otimes \mathcal{K}, \varepsilon^{m} \otimes_{*} \mathrm{id}\right)
$$


imprimitivity bimodule from Example 4.13, and let $X^{m}$ denote the associated $A^{m}-B^{m}$ imprimitivity bimodule as in Lemma 4.14 , with an $\left(A^{m} \otimes \mathcal{K}\right)-\left(B^{m} \otimes \mathcal{K}\right)$ imprimitivity bimodule isomorphism $\theta: X^{m} \otimes \mathcal{K} \rightarrow Y$. Then there is a unique $\delta^{m}-\varepsilon^{m}$ compatible coaction $\zeta^{m}$ on $X^{m}$ such that $\theta$ transports $\zeta^{m} \otimes_{*}$ id to $\eta$.

Proof. The diagram

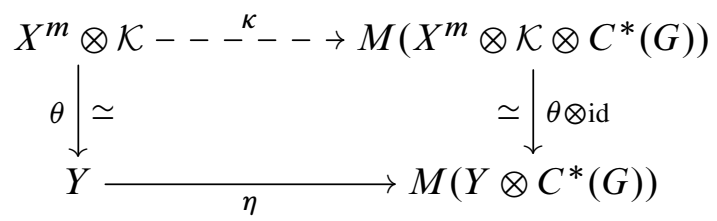

certainly has a unique commuting completion, and $\kappa$ is a $\left(\delta^{m} \otimes_{*}\right.$ id $)-\left(\varepsilon^{m} \otimes_{*}\right.$ id $)$ compatible coaction on $X^{m} \otimes \mathcal{K}$. In order to recognize that $\kappa$ is of the form $\zeta^{m} \otimes_{*}$ id, we need to know that, letting $\Sigma: \mathcal{K} \otimes C^{*}(G) \rightarrow C^{*}(G) \otimes \mathcal{K}$ be the flip isomorphism, for every $\xi \in X^{m}$, the element

$$
m:=\left(\operatorname{id}_{X^{m}} \otimes \Sigma\right) \circ(\theta \otimes \mathrm{id})^{-1} \circ \eta \circ \theta\left(\xi \otimes 1_{\mathcal{K}}\right)
$$

of the multiplier bimodule $M\left(X^{m} \otimes C^{*}(G) \otimes \mathcal{K}\right)$ is contained in the subset $M\left(X^{m} \otimes\right.$ $\left.C^{*}(G)\right) \otimes 1_{\mathcal{K}}$, and for this we need only check that for all $k \in \mathcal{K}$ we have

$$
\left(1_{A \otimes C^{*}(G)} \otimes k\right) \cdot m=m \cdot\left(1_{B \otimes C^{*}(G)} \otimes k\right) \in X^{m} \otimes C^{*}(G) \otimes \mathcal{K},
$$

which follows from the properties of the maps involved. Then it is routine to check that the resulting map $\xi^{m}$ is a $\delta^{m}-\varepsilon^{m}$ compatible coaction on $X^{m}$.

Definition 4.16. A coaction functor $\tau$ is Morita compatible if whenever $(X, \zeta)$ is an $(A, \delta)-(B, \varepsilon)$ imprimitivity bimodule, with associated $A^{m}-B^{m}$ imprimitivity bimodule $X^{m}$ as above, the Rieffel correspondence of ideals satisfies

$$
X^{m} \text {-Ind } B_{\tau}^{m}=A_{\tau}^{m} .
$$

We will use without comment the simple observation that if $(A, \delta)$ (and hence also $(B, \varepsilon))$ is maximal, then we can replace $X^{m}$ by $X$ and regard the natural surjection $q_{A}^{\tau}$ as going from $A$ to $A^{\tau}$ (and similarly for $B$ ), since the maximalizing maps $q_{A}^{m}$ and $q_{B}^{m}$ can be combined to give an isomorphism of the $A^{m}-B^{m}$ imprimitivity bimodule $X^{m}$ onto $X$.

Remark 4.17. Caution: Definition 4.16 is not a direct analogue of the definition of Morita compatibility in [Baum et al. 2016, Definition 3.2], but it suits our purposes in working with coaction functors, as we will see in Proposition 4.24.

Remark 4.18. Lemma 4.15 says in particular that maximalization preserves Morita equivalence of coactions. This is almost new: it also follows from first applying 
the cross-product functor, noting that the dual actions are "weakly proper $G \rtimes G$ algebras" in the sense of [Buss and Echterhoff 2014], then applying [Buss and Echterhoff 2015, Corollary 4.6] with the universal crossed-product norm (denoted by $u$ in [Buss and Echterhoff 2014]).

Lemma 4.19. A coaction functor $\tau$ is Morita compatible if and only if whenever $(X, \zeta)$ is an $(A, \delta)-(B, \varepsilon)$ imprimitivity bimodule, there are an $A^{\tau}-B^{\tau}$ imprimitivity bimodule $X^{\tau}$ and a $q_{A}^{\tau}-q_{B}^{\tau}$ compatible imprimitivity-bimodule homomorphism $q_{X}^{\tau}: X^{m} \rightarrow X^{\tau}$.

Proof. Given $X^{\tau}$ and $q_{X}^{\tau}$ with the indicated properties, by [Echterhoff et al. 2006, Lemma 1.20] we have

$$
X^{m} \text {-Ind } B_{\tau}^{m}=X^{m} \text {-Ind ker } q_{B}^{\tau}=\operatorname{ker} q_{A}^{\tau}=A_{\tau}^{m} .
$$

It follows that $\tau$ is Morita compatible.

Conversely, suppose $\tau$ is Morita compatible, and let $\left(X^{m}, \zeta^{m}\right)$ be as above. Then, by the Rieffel correspondence, $X^{\tau}:=X^{m} / X^{m} \cdot B_{\tau}^{m}$ is an $A^{m} / A_{\tau}^{m}-B^{m} / B_{\tau}^{m}$ imprimitivity bimodule, and the quotient map $q_{X}^{\tau}: X^{m} \rightarrow X^{\tau}$ is compatible with the quotient maps $A^{m} \mapsto A^{m} / A_{\tau}^{m}$ and $B^{m} \mapsto B_{\tau}^{m}$. Via the unique isomorphisms making the diagrams
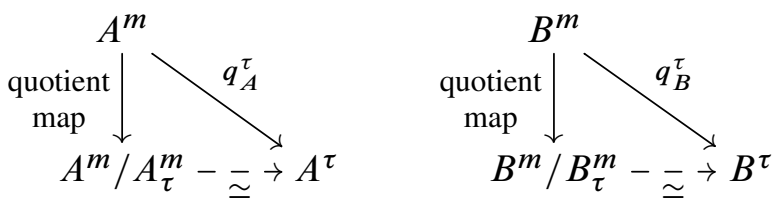

commute, $q_{X}^{\tau}$ becomes $q_{A}^{\tau}-q_{B}^{\tau}$ compatible.

Example 4.20. It follows trivially that the maximalization functor is Morita compatible.

Lemma 4.21. The identity functor on coactions is Morita compatible.

Proof. Let $(X, \zeta)$ be an $(A, \delta)-(B, \varepsilon)$ imprimitivity bimodule, and let $\left(X^{m}, \zeta^{m}\right)$ be the associated $\left(A^{m}, \delta^{m}\right)-\left(B^{m}, \varepsilon^{m}\right)$ imprimitivity bimodule from Lemma 4.15. By Lemma 4.19 it suffices to find a $q_{A}^{m}-q_{B}^{m}$ compatible imprimitivity-bimodule homomorphism $q_{X}^{m}: X^{m} \rightarrow X$. Now, $X^{m}$ is the upper right corner of the $2 \times 2$ matrix representation of the linking algebra $L^{m}$, and the maximalization map $q_{L}^{m}$ of the linking algebra $L$ of $X$ preserves the upper right corners. Thus $q_{L}^{m}$ takes $X^{m}$ onto $X$, and simple algebraic manipulations show that it has the right properties.

Theorem 4.22. The greatest lower bound of the collection of all exact and Morita compatible coaction functors is itself exact and Morita compatible. 
Proof. Let $\mathcal{T}$ be the collection of all exact and Morita compatible coaction functors, and let $\tau$ be the greatest lower bound of $\mathcal{T}$. As in the proof of Theorem 4.9, for every coaction $(A, \delta)$ we have

$$
A_{\tau}^{m}=\underset{\sigma \in \mathcal{T}}{\overline{\operatorname{span}}} A_{\sigma}^{m} .
$$

For exactness, we apply Definition 4.10. Let

$$
0 \longrightarrow(I, \gamma) \stackrel{\phi}{\longrightarrow}(A, \delta) \stackrel{\psi}{\longrightarrow}(B, \varepsilon) \longrightarrow 0
$$

be a short exact sequence of coactions. Then

$$
\begin{aligned}
& \phi^{m}\left(I_{\tau}^{m}\right)=\phi^{m}\left(\underset{\sigma \in \mathcal{T}}{\left.\operatorname{span}_{\sigma}^{m}\right)}\right. \\
& \left.=\underset{\sigma \in \mathcal{T}}{\operatorname{span}_{\sigma}^{m}} \phi^{m}\right) \\
& =\underset{\sigma \in \mathcal{T}}{\operatorname{\operatorname {span}}}\left(\phi^{m}\left(I^{m}\right) \cap A_{\sigma}^{m}\right) \quad \text { (since } \sigma \text { is exact) } \\
& =\phi^{m}\left(I^{m}\right) \cap \overline{\operatorname{span}}_{\sigma \in \mathcal{T}} A_{\sigma}^{m}
\end{aligned}
$$

(since all spaces involved are ideals in $C^{*}$-algebras)

$$
=\phi^{m}\left(I^{m}\right) \cap A_{\tau}^{m},
$$

and

$$
\begin{aligned}
\phi^{m}\left(I^{m}\right)+A_{\tau}^{m} & =\phi^{m}\left(I^{m}\right)+\underset{\sigma \in \mathcal{T}}{\overline{\operatorname{span}}} A_{\sigma}^{m} \\
& =\underset{\sigma \in \mathcal{T}}{\overline{\operatorname{span}}}\left(\phi^{m}\left(I^{m}\right)+A_{\sigma}^{m}\right) \\
& \left.=\underset{\sigma \in \mathcal{T}}{\overline{\operatorname{span}}}\left(\psi^{m}\right)^{-1}\left(B_{\sigma}^{m}\right) \quad \text { (since } \sigma \text { is exact }\right) \\
& =\left(\psi^{m}\right)^{-1}\left(\underset{\sigma \in \mathcal{T}}{\left.\operatorname{span}_{\sigma}\right)}\right. \\
& =\left(\psi^{m}\right)^{-1}\left(B_{\tau}^{m}\right),
\end{aligned}
$$

so $\tau$ is exact.

For Morita compatibility, let $(X, \zeta)$ be an $(A, \delta)-(B, \varepsilon)$ imprimitivity bimodule, with associated $A^{m}-B^{m}$ imprimitivity bimodule $X^{m}$. Then

$$
\begin{aligned}
X^{m} \text {-Ind } B_{\tau}^{m} & =X^{m} \text {-Ind } \overline{\sigma \in \mathcal{T}} B_{\sigma}^{m} \\
& =\overline{\operatorname{span}}_{\sigma \in \mathcal{T}} X^{m} \text {-Ind } B_{\sigma}^{m} \quad \text { (by continuity of Rieffel induction) } \\
& =\overline{\operatorname{san}}_{\sigma \in \mathcal{T}} A_{\sigma}^{m} \quad \text { (since } \sigma \text { is Morita compatible) } \\
& =A_{\tau}^{m},
\end{aligned}
$$

so $\tau$ is Morita compatible. 
Definition 4.23. We call the above greatest lower bound of the collection of all exact and Morita compatible coaction functors the minimal exact and Morita compatible coaction functor.

Comparison with [Baum et al. 2016]. As we mentioned previously, [Baum et al. 2016, p. 8] defines one crossed-product functor $\sigma_{1}$ to be smaller than another one $\sigma_{2}$, written $\sigma_{1} \leq \sigma_{2}$, if the natural surjection $A \rtimes_{\alpha, \sigma_{2}} G \rightarrow A \rtimes_{\alpha, r} G$ factors through the $\sigma_{1}$-crossed product.

Let $\tau$ be a coaction functor, and let $\sigma=\tau \circ \mathrm{CP}$ be the associated crossed-product functor, i.e.,

$$
(A, \alpha)^{\sigma}=A \rtimes_{\alpha, \sigma} G:=\left(A \rtimes_{\alpha} G\right)^{\tau} .
$$

For a morphism $\phi:(A, \alpha) \rightarrow(B, \beta)$ of actions, we write

$$
\phi \rtimes_{\sigma} G=(\phi \rtimes G)^{\tau}: A \rtimes_{\alpha, \sigma} G \rightarrow B \rtimes_{\beta, \sigma} G
$$

for the associated morphism of $\sigma$-crossed products.

Proposition 4.24. With the above notation, if the coaction functor $\tau$ is exact or Morita compatible, then the associated crossed-product functor $\sigma$ has the same property. Moreover, if $\tau_{1} \leq \tau_{2}$ then $\sigma_{1} \leq \sigma_{2}$.

Proof. The last statement follows immediately from the definitions. For the other statement, first assume that $\tau$ is exact, and let

$$
0 \longrightarrow(I, \gamma) \stackrel{\phi}{\longrightarrow}(A, \alpha) \stackrel{\psi}{\longrightarrow}(B, \beta) \longrightarrow 0
$$

be a short exact sequence of actions. Then the sequence

$$
0 \longrightarrow\left(I \rtimes_{\gamma} G, \hat{\gamma}\right) \stackrel{\phi \rtimes G}{\longrightarrow}\left(A \rtimes_{\alpha} G, \widehat{\alpha}\right) \stackrel{\psi \rtimes G}{\longrightarrow}\left(B \rtimes_{\beta} G, \widehat{\beta}\right) \longrightarrow 0
$$

of coactions is exact, since the full-crossed-product functor is exact. Then by exactness of $\tau$ we see that the sequence

$$
0 \longrightarrow I \rtimes_{\gamma, \sigma} G \stackrel{\phi \rtimes_{\sigma} G}{\longrightarrow} A \rtimes_{\alpha, \sigma} G \stackrel{\psi \rtimes_{\sigma} G}{\longrightarrow} B \rtimes_{\beta, \sigma} G \longrightarrow 0
$$

is also exact.

On the other hand, assume that the coaction functor $\tau$ is Morita compatible. As in [Baum et al. 2016, Section 3], the unwinding isomorphism $\Phi$, which is the integrated form of the covariant pair

$$
\begin{aligned}
\pi(a \otimes k) & =i_{A}(a) \otimes k, \\
u_{s} & =i_{G}(s) \otimes \lambda_{s},
\end{aligned}
$$


fits into a diagram

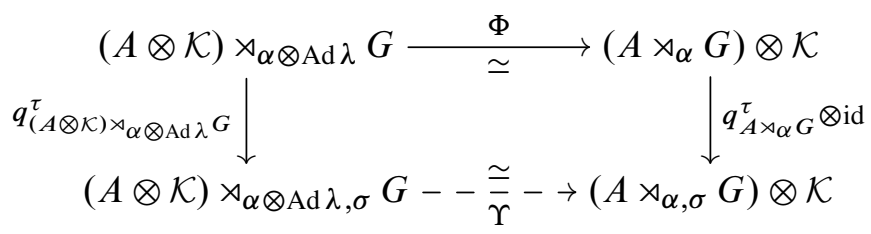

i.e.,

$$
\operatorname{ker} q_{(A \otimes \mathcal{K}) \rtimes_{\alpha \otimes \mathrm{Ad} \lambda} G}^{\tau}=\operatorname{ker}\left(q_{A \rtimes_{\alpha} G}^{\tau} \otimes \mathrm{id}\right) \circ \Phi .
$$

The diagram (4-6) fits into a more elaborate diagram

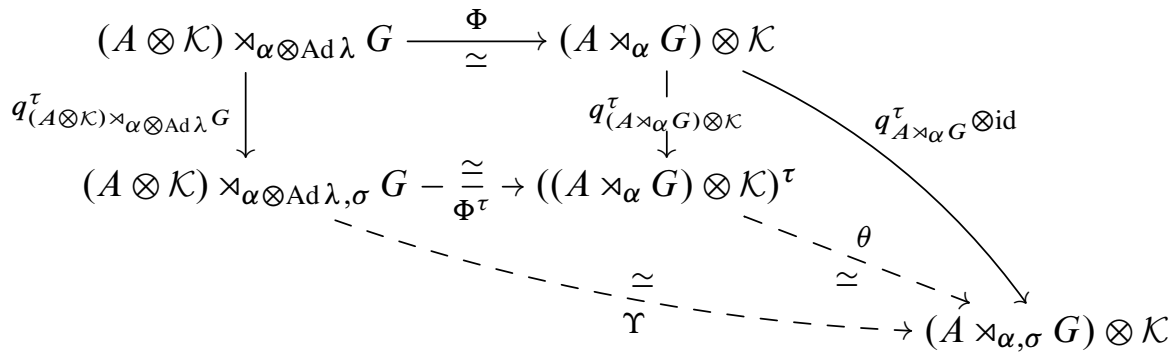

which we proceed to analyze. There is a unique

$$
(\widehat{\alpha \otimes \operatorname{Ad} \lambda})^{\tau}-\left(\widehat{\alpha} \otimes_{*} \mathrm{id}\right)^{\tau}
$$

equivariant homomorphism $\Phi^{\tau}$ making the upper left rectangle commute, since $\tau$ is functorial. Moreover, $\Phi^{\tau}$ is an isomorphism since $\Phi$ is, again by functoriality. Applying Morita compatibility of $\tau$ to the equivariant $\left(\left(A \rtimes_{\alpha} G\right) \otimes \mathcal{K}\right)-\left(A \rtimes_{\alpha} G\right)$ imprimitivity bimodule $\left(A \rtimes_{\alpha} G\right) \otimes L^{2}(G)$ shows that there is a unique

$$
\left(\widehat{\alpha} \otimes_{*} \mathrm{id}\right)^{\tau}-\left(\hat{\alpha}^{\tau} \otimes_{*} \mathrm{id}\right)
$$

equivariant isomorphism $\theta$ that makes the upper right triangle commute. Thus there is a unique isomorphism $\Upsilon$ making the lower left triangle commute, and then the outer quadrilateral commutes, as desired.

Question 4.25. (1) Is the minimal exact and Morita compatible crossed product of [Baum et al. 2016, Section 4] naturally isomorphic to the composition of the minimal exact and Morita compatible coaction functor and the full crossed product?

(2) More generally, given a crossed-product functor on actions, when does it decompose as a full crossed product followed by a coaction functor? Does it make any difference if the crossed-product functor is exact or Morita compatible? 


\section{Decreasing coaction functors}

In this section we introduce a particular type of coaction functor with the convenient property that we do not need to check things by going through the maximalization functor, as we'll see in Propositions 5.4 and 5.5. Suppose that for each coaction $(A, \delta)$ we have a coaction $\left(A^{\tau}, \delta^{\tau}\right)$ and a $\delta-\delta^{\tau}$ equivariant surjection $Q^{\tau}: A \rightarrow A^{\tau}$, and further suppose that for each morphism $\phi:(A, \delta) \rightarrow(B, \varepsilon)$ we have

$$
\operatorname{ker} Q_{A}^{\tau} \subset \operatorname{ker} Q_{B}^{\tau} \circ \phi \text {, }
$$

so that there is a unique morphism $\phi^{\tau}$ making the diagram

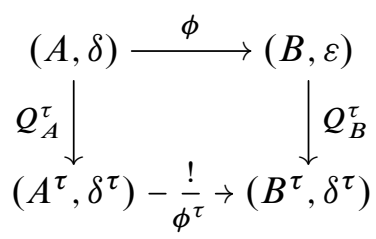

commute. The uniqueness and surjectivity assumptions imply that $\tau$ constitutes a functor on the category of coactions, and moreover $Q^{\tau}$ : id $\rightarrow \tau$ is a natural transformation.

Definition 5.1. We call a functor $\tau$ as above decreasing if for each coaction $(A, \delta)$ we have

$$
\operatorname{ker} Q_{A}^{\tau} \subset \operatorname{ker} \Lambda_{A}
$$

Lemma 5.2. Every decreasing functor $\tau$ on coactions is a coaction functor, and moreover $\tau \leq \mathrm{id}$.

Proof. For each coaction $(A, \delta)$, define a homomorphism $q_{A}^{\tau}$ by the commutative diagram

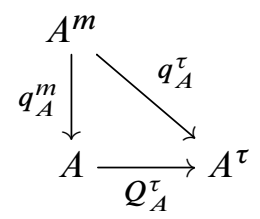

where $q_{A}^{m}$ is the maximalization map. The map $q^{\tau}$ is natural and surjective since both $q^{m}$ and $Q^{\tau}$ are. We have

$$
\begin{aligned}
\operatorname{ker} q_{A}^{\tau} & =\left\{a \in A^{m}: q_{A}^{m}(a) \in \operatorname{ker} Q_{A}^{\tau}\right\} \\
& \subset\left\{a \in A^{m}: q_{A}^{m}(a) \in \operatorname{ker} \Lambda_{A}\right\} \\
& =\operatorname{ker} \Lambda_{A} \circ q_{A}^{m} \\
& =\operatorname{ker} \Lambda_{A} m
\end{aligned}
$$

Thus $\tau$ is a coaction functor, and then $\tau \leq$ id by Lemma 4.8. 
Notation 5.3. For a decreasing coaction functor $\tau$ and any coaction $(A, \delta)$ put

$$
A_{\tau}=\operatorname{ker} Q_{A}^{\tau} \text {. }
$$

Proposition 5.4. A decreasing coaction functor $\tau$ is exact if and only if for any short exact sequence

$$
0 \longrightarrow\left(I, \delta_{I}\right) \stackrel{\phi}{\longrightarrow}(A, \delta) \stackrel{\psi}{\longrightarrow}\left(B, \delta^{I}\right) \longrightarrow 0
$$

of coactions, both

$$
\phi\left(I_{\tau}\right)=\phi(I) \cap A_{\tau}
$$

and

$$
\phi(I)+A_{\tau} \supset \psi^{-1}\left(B_{\tau}\right)
$$

hold.

Proof. The proof is very similar to, and slightly easier than, that of Theorem 4.12, using the commutative diagram

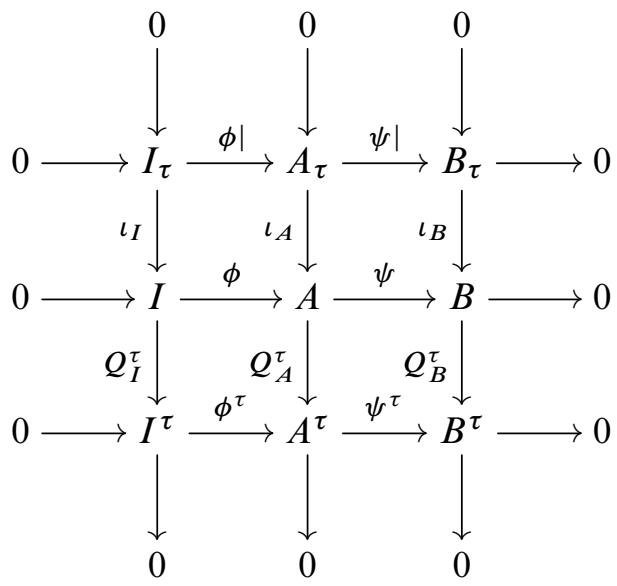

Proposition 5.5. A decreasing coaction functor $\tau$ is Morita compatible if and only if whenever $(X, \zeta)$ is an $(A, \delta)-(B, \varepsilon)$ imprimitivity bimodule, there are an $A^{\tau}-B^{\tau}$ imprimitivity bimodule $X^{\tau}$ and a $Q_{A}^{\tau}-Q_{B}^{\tau}$ compatible imprimitivity-bimodule homomorphism $Q_{X}^{\tau}: X \rightarrow X^{\tau}$.

Proof. First suppose $\tau$ is Morita compatible. Let $(X, \zeta)$ be an $(A, \delta)-(B, \varepsilon)$ imprimitivity bimodule, and let $q_{X}^{\tau}: X^{m} \rightarrow X^{\tau}$ be a $q_{A}^{m}-q_{B}^{m}$ compatible imprimitivitybimodule homomorphism onto an $A^{\tau}-B^{\tau}$ imprimitivity bimodule $X^{\tau}$, as in Lemma 4.19. By Lemmas 4.21 and 4.19 there is also a $q_{A}^{m}-q_{B}^{m}$ compatible imprimitivity bimodule homomorphism $q_{X}^{m}$ of $X^{m} \rightarrow X$. By definition, we have

$$
q_{A}^{\tau}=Q_{A}^{\tau} \circ q_{A}^{m}: A^{m} \rightarrow A^{\tau} .
$$


Thus

$$
\begin{aligned}
\operatorname{ker} q_{X}^{m} & =\left(\operatorname{ker} q_{A}^{m}\right) \cdot X^{m} \\
& \subset\left(\operatorname{ker} Q_{A}^{\tau} \circ q_{A}^{m}\right) \cdot X^{m} \\
& =\left(\operatorname{ker} q_{A}^{\tau}\right) \cdot X^{m} \\
& =\operatorname{ker} q_{X}^{\tau},
\end{aligned}
$$

and hence $q_{X}^{\tau}$ factors through a commutative diagram

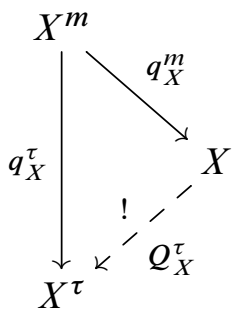

for a unique imprimitivity bimodule homomorphism $Q_{X}^{\tau}$. Moreover, $Q_{X}^{\tau}$ is compatible on the left with $Q_{A}^{\tau}$ by construction, and similar reasoning, using the Rieffel correspondence of ideals, shows that it is also $Q_{B}^{\tau}$ compatible on the right.

Conversely, suppose we have $(X, \zeta), X^{\tau}$ and $Q_{X}^{\tau}$ as indicated, and let $\left(X^{m}, \zeta^{m}\right)$ be the associated $\left(A^{m}, \delta^{m}\right)-\left(B^{m}, \varepsilon^{m}\right)$ imprimitivity bimodule from Lemma 4.15. By Lemma 4.19 it suffices to find a $q_{A}^{m}-q_{B}^{m}$ compatible imprimitivity-bimodule homomorphism $q_{X}^{\tau}: X^{m} \rightarrow X^{\tau}$. Since $q^{\tau}=Q^{\tau} \circ q^{m}$ on both $A^{m}$ and $B^{m}$, by Lemma 4.21 and our assumptions we can take $q_{X}^{\tau}=Q_{X}^{\tau} \circ q_{X}^{m}$.

\section{Coaction functors from large ideals}

The most important source of examples of the decreasing coaction functors of the preceding section is large ideals. We recall some basic concepts from [KLQ 2013; 2016]. Let $E$ be an ideal of $B(G)$ that is large, meaning it is nonzero, $G$-invariant, and weak*-closed. Then the preannihilator ${ }^{\perp} E$ of $E$ in $C^{*}(G)$ is an ideal contained in the kernel of the regular representation $\lambda$. Write $C_{E}^{*}(G)=C^{*}(G) /{ }^{\perp} E$ for the quotient group $C^{*}$-algebra and $q_{E}: C^{*}(G) \rightarrow C_{E}^{*}(G)$ for the quotient map. The ideal ${ }^{\perp} E=\operatorname{ker} q_{E}$ of $C^{*}(G)$ is weakly $\delta_{G}$-invariant, i.e., $\delta_{G}$ descends to a coaction, which we denote by $\delta_{G}^{E}$, on the quotient $C_{E}^{*}(G)$.

For any coaction $(A, \delta)$ and any large ideal $E$ of $B(G)$,

$$
A_{E}:=\{a \in A: E \cdot a=\{0\}\}=\operatorname{ker}\left(\mathrm{id} \otimes q_{E}\right) \circ \delta
$$

is a small ideal of $A$ (that is, an ideal contained in $\operatorname{ker} j_{A}=\operatorname{ker} \Lambda_{A}$ ) and we write $A^{E}=A / A_{E}$ for the quotient $C^{*}$-algebra and $Q_{A}^{E}: A \rightarrow A^{E}$ for the quotient 
map. $A_{E}$ is weakly $\delta$-invariant [KLQ 2016, Lemma 3.5], and we write $\delta^{E}$ for the quotient coaction on $A^{E}$.

Remark 6.1. The properties of the $B(G)$-module structure (see the Appendix) allow for a shorter proof of invariance than in [KLQ 2016]: if $a \in A_{E}, f \in B(G)$, and $g \in E$ then

$$
g \cdot(f \cdot a)=(g f) \cdot a=0,
$$

because $E$ is an ideal, and it follows that $B(G) \cdot A_{E} \subset A_{E}$.

Proposition 6.2. The functor $(A, \delta) \mapsto\left(A^{E}, \delta^{E}\right)$ is a decreasing coaction functor, which we denote by $\tau_{E}$.

Proof. By the above discussion and Lemma 5.2, it suffices to observe that for any morphism $\phi:(A, \delta) \rightarrow(B, \varepsilon)$ of coactions and for all $a \in \operatorname{ker} Q_{A}^{E}$ and $f \in E$,

$$
f \cdot \phi(a)=\phi(f \cdot a)=0,
$$

which implies that $\operatorname{ker} Q_{A}^{E} \subset \operatorname{ker} Q_{B}^{E} \circ \phi$.

Remark 6.3. Proposition 6.2 should be compared with [Buss and Echterhoff 2014, Corollary 6.5 and Lemma 7.1], [Buss and Echterhoff 2015, Lemma 2.3], and [Baum et al. 2016, Lemma A.3].

Example 6.4. The functor $\tau_{B(G)}$ is the identity functor.

Example 6.5. The functor $\tau_{B_{r}(G)}$ is naturally isomorphic to the normalization functor.

Example 6.6. The maximalization functor is not of the form $(A, \delta) \mapsto\left(A^{E}, \delta^{E}\right)$ for any large ideal $E$ of $B(G)$, because the maximalization functor is not decreasing in the sense of Definition 5.1.

Proposition 6.7. For a large ideal $E$ of $B(G)$, the coaction functor $\tau_{E}$ is exact if and only if, for every coaction $(A, \delta)$ and every strongly invariant ideal I of $A$,

$$
I+A_{E} \supset\{a \in A: E \cdot a \subset I\} .
$$

Proof. Let

$$
0 \longrightarrow(I, \zeta) \stackrel{\phi}{\longrightarrow}(A, \delta) \stackrel{\psi}{\longrightarrow}(B, \varepsilon) \longrightarrow 0
$$

be a short exact sequence of coactions. Exactness of the associated sequence

$$
0 \longrightarrow I^{E} \stackrel{\phi^{E}}{\longrightarrow} A^{E} \stackrel{\psi^{E}}{\longrightarrow} B^{E} \longrightarrow 0
$$

will not be affected if we replace the short exact sequence (6-2) by an isomorphic one, so without loss of generality $\phi$ is the inclusion of an ideal $I$ of $A$ and $\psi$ is the 
quotient map onto $B=A / I$. By Proposition 5.4, the sequence (6-3) is exact if and only if

$$
I_{E}=I \cap A_{E}
$$

and

$$
I+A_{E} \supset \psi^{-1}\left(B_{E}\right) .
$$

Since

$$
I_{E}=\{a \in I: E \cdot a=\{0\}\}
$$

(6-4) automatically holds in this context. On the other hand, (6-5) is equivalent to (6-1) because

$$
\begin{aligned}
B_{E} & =\{a+I \in B=A / I: E \cdot(a+I)=\{0\}\} \\
& =\{a+I: E \cdot a \subset I\} .
\end{aligned}
$$

Remark 6.8. Techniques similar to those used in the above proof, showing that (6-4) holds automatically, can also be used to show that the functor $\tau_{E}$ preserves injectivity of morphisms: if $\phi: A \rightarrow B$ is an injective equivariant homomorphism and $a \in \operatorname{ker} \phi^{E}$, then we can write $a=Q_{A}^{E}\left(a^{\prime}\right)$ for some $a^{\prime} \in A$. We have

$$
0=\phi^{E}(a)=\phi^{E} \circ Q_{A}^{E}\left(a^{\prime}\right)=Q_{B}^{E} \circ \phi\left(a^{\prime}\right),
$$

so

$$
\phi(a) \in \operatorname{ker} Q_{B}^{E}=B_{E} .
$$

Thus for all $f \in E$ we have

$$
0=f \cdot \phi\left(a^{\prime}\right)=\phi\left(f \cdot a^{\prime}\right),
$$

so $f \cdot a^{\prime}=0$ since $\phi$ is injective. But then $a^{\prime} \in A_{E}=\operatorname{ker} Q_{A}^{E}$, so $a=0$. This remark should be compared with [Buss and Echterhoff 2014, Proposition 6.2].

Corollary 6.9. Let $E$ and $F$ be large ideals of $B(G)$, and let $\langle E F\rangle$ denote the weak ${ }^{*}$-closed linear span of the set $E F$ of products. If $\tau_{E}$ or $\tau_{F}$ is exact then $\langle E F\rangle=E \cap F$.

Proof. Without loss of generality assume that $\tau_{E}$ is exact. Note that, since $E$ is an ideal of $B(G)$,

$$
{ }^{\perp} E=\left\{a \in C^{*}(G): E \cdot a=\{0\}\right\},
$$

and similarly for ${ }^{\perp} F$. We claim that

$$
{ }^{\perp} E+{ }^{\perp} F={ }^{\perp}\langle E F\rangle .
$$


To see this, note that, since $E$ is exact, by Proposition 6.7 with $(A, \delta)=\left(C^{*}(G), \delta_{G}\right)$ and $I={ }^{\perp} F$ we have

$$
{ }^{\perp} F+{ }^{\perp} E \supset\left\{a \in C^{*}(G): E \cdot a \subset{ }^{\perp} F\right\} .
$$

Now, for $a \in C^{*}(G)$ we have

$$
\begin{aligned}
E \cdot a \subset{ }^{\perp} F & \Longleftrightarrow F \cdot(E \cdot a)=\{0\} \\
& \Longleftrightarrow(E F) \cdot a=\{0\} \\
& \Longleftrightarrow\langle E F\rangle \cdot a=\{0\} \\
& \Longleftrightarrow a \in{ }^{\perp}\langle E F\rangle,
\end{aligned}
$$

where the equivalence at $*$ holds since for every $a \in C^{*}(G)$ the map from $B(G)$ to $C^{*}(G)$ defined by $f \mapsto f \cdot a$ is weak*-weak continuous. Thus ${ }^{\perp} F+{ }^{\perp} E \supset^{\perp}\langle E F\rangle$.

For the reverse containment, note that $E F \subset E$ because $E$ is an ideal, so $\langle E F\rangle \subset E$ because $E$ is weak*-closed, and hence ${ }^{\perp} E \subset{ }^{\perp}\langle E F\rangle$. Similarly, ${ }^{\perp} F \subset{ }^{\perp}\langle E F\rangle$, and so ${ }^{\perp} E+{ }^{\perp} F \subset{ }^{\perp}\langle E F\rangle$, proving the claim.

Now, since ${ }^{\perp} E$ and ${ }^{\perp} F$ are closed ideals of $C^{*}(G)$, it follows from the elementary duality theory for Banach spaces that

$$
{ }^{\perp} E+{ }^{\perp} F={ }^{\perp}(E \cap F),
$$

and the corollary follows upon taking annihilators.

The following result should be compared with [Baum et al. 2016, Lemma A.5]: Proposition 6.10. The coaction functor $\tau_{E}$ is Morita compatible.

Proof. Let $(X, \zeta)$ be an $(A, \delta)-(B, \varepsilon)$ imprimitivity bimodule. Since $\tau$ is decreasing, by Proposition 5.5, it suffices to show that $X$-Ind $B_{E}=A_{E}$. The external tensor product $X \otimes C_{E}^{*}(G)$ is an $\left(A \otimes C_{E}^{*}(G)\right)-\left(B \otimes C_{E}^{*}(G)\right)$ imprimitivity bimodule, and we have an $\left(\mathrm{id}_{A} \otimes q_{E}\right)-\left(\mathrm{id}_{B} \otimes q_{E}\right)$ compatible imprimitivity bimodule homomorphism

$$
\operatorname{id}_{X} \otimes q_{E}: X \otimes C^{*}(G) \rightarrow X \otimes C_{E}^{*}(G)
$$

The composition

$$
\left(\operatorname{id}_{X} \otimes q_{E}\right) \circ \zeta: X \rightarrow M\left(X \otimes C_{E}^{*}(G)\right)
$$

is an $\left(\mathrm{id}_{A} \otimes q_{E}\right) \circ \delta-\left(\mathrm{id}_{B} \otimes q_{E}\right) \circ \varepsilon$ compatible imprimitivity bimodule homomorphism. We have

$$
\begin{aligned}
& \operatorname{ker}\left(\operatorname{id}_{A} \otimes q_{E}\right) \circ \delta=A_{E}, \\
& \operatorname{ker}\left(\operatorname{id}_{B} \otimes q_{E}\right) \circ \varepsilon=B_{E} .
\end{aligned}
$$


Thus, by [Echterhoff et al. 2006, Lemma 1.20], $A_{E}$ is the ideal of $A$ associated to the ideal $B_{E}$ of $B$ via the Rieffel correspondence.

Remark 6.11. Proposition 6.10 subsumes [KLQ 2016, Lemma 4.8], which is the special case of exterior equivalent coactions. It is tempting to try to use this to simplify the proof of [loc. cit., Theorem 4.6], which says that $(A, \delta)$ satisfies $E$ crossed-product duality if and only if it is isomorphic to $\left(A^{m E}, \delta^{m E}\right)$, since we have Morita equivalences

$$
\left(A^{m}, \delta^{m}\right) \sim_{M}\left(A^{m} \otimes \mathcal{K}, \delta \otimes_{*} \text { id }\right) \sim_{M}\left(A \rtimes_{\delta} G \rtimes_{\widehat{\delta}} G, \widehat{\hat{\delta}}\right) .
$$

However, it turns out that appealing to Proposition 6.10 would not shorten the proof much. Nevertheless, it is interesting to note that, by Proposition 6.10, we have

$$
(A, \delta)=\left(A^{m E}, \delta^{m E}\right) \Longleftrightarrow\left(A \otimes \mathcal{K}, \delta \otimes_{*} \mathrm{id}\right)=\left(\left(A^{m} \otimes \mathcal{K}\right)^{E},\left(\delta^{m} \otimes_{*} \mathrm{id}\right)^{E}\right),
$$

or, equivalently,

$$
\operatorname{ker} \Phi=\left(A \rtimes_{\delta} G \rtimes_{\hat{\delta}} G\right)_{E},
$$

which by definition is equivalent to $E$-crossed-product duality for $(A, \delta)$.

For some purposes, albeit not for the purposes of this paper, a more appropriate coaction functor associated to $E$ is the following (see also [Buss and Echterhoff 2014, Theorem 5.1]):

Definition 6.12. The E-ization of a coaction $(A, \delta)$ is

$$
\left(A^{E \text {-ize }}, \delta^{E \text {-ize }}\right):=\left(\left(A^{m}\right)^{E},\left(\delta^{m}\right)^{E}\right) .
$$

$E$-ization is a functor on the category of coactions, being the composition of the functors maximalization and $\tau_{E}$. The $E$-ization of a $\delta-\varepsilon$ equivariant homomorphism $\phi: A \rightarrow B$ is

$$
\phi^{E \text {-ize }}=\left(\phi^{m}\right)^{E}: A^{m E} \rightarrow B^{m E} .
$$

Proposition 6.13. E-ization is a coaction functor.

Proof. We must produce a suitable natural transformation $q^{E \text {-ize }}:\left(A^{m}, \delta^{m}\right) \rightarrow$ $\left(A^{E \text {-ize }}, \delta^{E \text {-ize }}\right)$, and we take

$$
q_{A}^{E \text {-ize }}=Q_{A^{m}}^{E}: A^{m} \rightarrow A^{m E}=A^{E \text {-ize }} .
$$

The map $q^{E \text {-ize }}$ is natural since $\tau_{E}$ is a decreasing coaction functor.

Theorem 6.14. For any large ideal $E$ of $B(G)$, the E-ization coaction functor is Morita compatible. 
Proof. Let $(X, \zeta)$ be an $(A, \delta)-(B, \varepsilon)$ imprimitivity bimodule, with associated $\left(A^{m}, \delta^{m}\right)-\left(B^{m}, \varepsilon^{m}\right)$ imprimitivity bimodule $\left(X^{m}, \zeta^{m}\right)$. We must show that

$$
X^{m} \text {-Ind } \operatorname{ker} q_{B}^{E \text {-ize }}=\operatorname{ker} q_{A}^{E \text {-ize }} .
$$

But this follows immediately by applying Proposition 6.10 to $\left(X^{m}, \zeta^{m}\right)$, since

$$
q_{A}^{E \text {-ize }}=Q_{E}^{A^{m}} \quad \text { and } \quad q_{B}^{E \text {-ize }}=Q_{E}^{B^{m}} .
$$

Remark 6.15. For any large ideal $E$, the two coaction functors $\tau_{E}$ and $E$-ization have similar properties; e.g., they are both Morita compatible (Proposition 6.10 and Theorem 6.14). However, in general they are not naturally isomorphic functors. For example, if $E=B(G)$ then $\tau_{E}$ is the identity functor and $E$-ization is maximalization. That being said, for $E=B_{r}(G)$ we do have $\tau_{E} \cong \tau_{E} \circ$ maximalization.

Note that, given a coaction $(A, \delta)$, we have two homomorphisms of the maximalization $\left(A^{m}, \delta^{m}\right)$ :

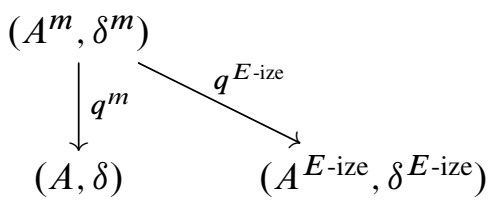

In [KLQ 2013, Definition 3.7] we said $(A, \delta)$ is E-determined from its maximalization if $\operatorname{ker} q^{m}=\operatorname{ker} q^{E \text {-ize }}$, in which case there is a natural isomorphism $(A, \delta) \cong\left(A^{E \text {-ize }}, \delta^{E \text {-ize }}\right)$.

Given an action $(B, \alpha)$, in [KLQ 2013, Definition 6.1] we defined the $E$-crossed product as

$$
B \rtimes_{\alpha, E} G=\left(B \rtimes_{\alpha} G\right) /\left(B \rtimes_{\alpha} G\right)_{E}=\left(B \rtimes_{\alpha} G\right)^{E},
$$

where in the last expression we have composed the full-crossed-product functor with $\tau_{E}$.

As in [Buss and Echterhoff 2014, Definition 4.5], we say a coaction $(A, \delta)$ satisfies $E$-duality (called " $E$-crossed product duality" in [KLQ 2016, Definition 4.3]), or is an E-coaction, if there is an isomorphism $\theta$ making the diagram

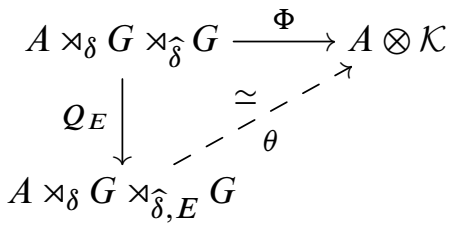

commute, or, equivalently,

$$
\operatorname{ker} \Phi=\left(A \rtimes_{\delta} G \rtimes_{\hat{\delta}} G\right)_{E},
$$

where $\Phi$ is the canonical surjection. 
In [KLQ 2016, Theorem 4.6] we proved that $(A, \delta)$ is an $E$-coaction if and only if it is $E$-determined from its maximalization. (Theorem 5.1 of [Buss and Echterhoff 2014] proves the converse direction.)

Lemma 6.16. For a coaction $(A, \delta)$, the following are equivalent:

(1) $(A, \delta)$ is an E-coaction.

(2) $(A, \delta)$ is $E$-determined from its maximalization.

(3) There exists a maximal coaction $(B, \varepsilon)$ such that $(A, \delta) \cong\left(B^{E}, \varepsilon^{E}\right)$.

Proof. The equivalence of (1) and (2) is [KLQ 2016, Theorem 4.6], and (2) trivially implies (3). Assume (3), i.e., that $(B, \varepsilon)$ is maximal and we have an isomorphism $\theta:\left(B^{E}, \varepsilon^{E}\right) \rightarrow(A, \delta)$. The surjection $Q_{E}^{B}:(B, \varepsilon) \rightarrow\left(B^{E}, \varepsilon^{E}\right)$ is a maximalization, since $\varepsilon$ is maximal and $\operatorname{ker} Q_{E}^{B} \subset \operatorname{ker} q_{B}^{n}$. Thus $\theta \circ Q_{E}^{B}$ is a maximalization of $(A, \delta)$. Since any two maximalizations of $(A, \delta)$ are isomorphic, there is an isomorphism $\psi$ making the diagram

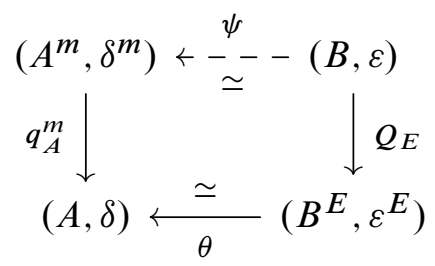

commute. Thus $q_{A}^{m} \circ \psi$ is also a maximalization of $(A, \delta)$. Therefore

$$
\operatorname{ker} q_{A}^{m}=\psi\left(\operatorname{ker} Q_{E}\right)=\psi\left(B_{E}\right)=A_{E}^{m},
$$

giving (2).

Theorem 6.17. The functor $\tau_{E}$ restricts to give an equivalence of the category of maximal coactions to the category of E-coactions.

In this statement, we mean the full subcategories of the category of coactions.

Proof. By abstract nonsense, it suffices to show that the functor is essentially surjective and fully faithful, i.e.,

(1) every $E$-coaction $(A, \delta)$ is isomorphic to $\left(B^{E}, \varepsilon^{E}\right)$ for some maximal coaction $(B, \varepsilon)$, and

(2) for any two maximal coactions $(A, \delta)$ and $(B, \varepsilon)$,

$$
\phi \mapsto \phi^{E}
$$

maps the set of equivariant homomorphisms $\phi: A \rightarrow B$ bijectively onto the set of equivariant homomorphisms $\psi: A^{E} \rightarrow B^{E}$. 
Statement (1) is immediate from Lemma 6.16. For (2), given maximal coactions $(A, \delta)$ and $(B, \varepsilon)$ and distinct nondegenerate equivariant homomorphisms $\phi, \psi: A \rightarrow B$, we have an equivariant commutative diagram

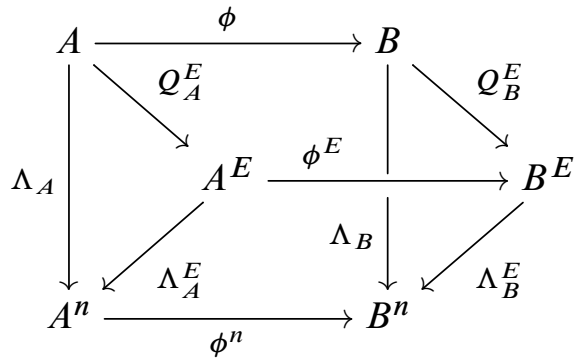

where $Q_{A}^{E}$ is a maximalization of $\left(A^{E}, \delta^{E}\right), \Lambda_{A}$ is a normalization of $(A, \delta)$, and $\Lambda_{A}^{E}$ is a normalization of $\left(A^{E}, \delta^{E}\right)$, and similarly for the right-hand triangle involving the $B \mathrm{~s}$. There is a similar commutative diagram for $\psi$. Since the normalizations $\phi^{n}$ and $\psi^{n}$ are distinct, by [Bédos et al. 2011, Corollary 6.1.19], we must have $\phi^{E} \neq \psi^{E}$ by commutativity of the diagram. This proves injectivity. For the surjectivity, let $\sigma: A^{E} \rightarrow B^{E}$ be an equivariant homomorphism. Then the maximalization $\sigma^{m}: A \rightarrow B$ of $\sigma$ is the unique equivariant homomorphism making the diagram

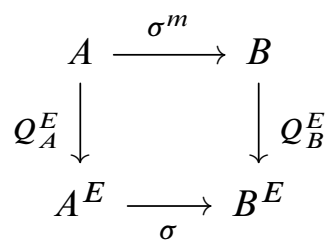

commute. Applying the functor $\tau_{E}$, we see that the diagram

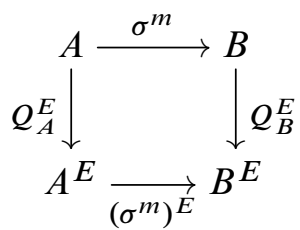

also commutes, so we must have $\sigma^{m}=\left(\left(\sigma^{m}\right)^{E}\right)^{m}$ by the universal property of maximalization, and hence $\sigma=\left(\sigma^{m}\right)^{E}$ by [loc. cit.].

Remark 6.18. Much of the development in this paper regarding "classical" categories carries over to the "nondegenerate" categories (involving multiplier algebras). The nondegenerate version of the above result resembles the "maximal-normal equivalence" of [Kaliszewski and Quigg 2009, Theorem 3.3], which says that normalization restricts to an equivalence between maximal and normal coactions. 
However, there are some properties missing: for example, the functor $\tau_{E}$ is not a reflector in the categorical sense, because

$$
Q_{E}:\left(A^{E}, \delta^{E}\right) \rightarrow\left(A^{E E}, \delta^{E E}\right)
$$

is not an isomorphism in general. Indeed, [KLQ 2016, Proposition 8.4] shows that if $(A, \delta)$ is a maximal coaction then the composition (id $\left.\otimes q_{E}\right) \circ \delta^{E}$ in the commutative diagram

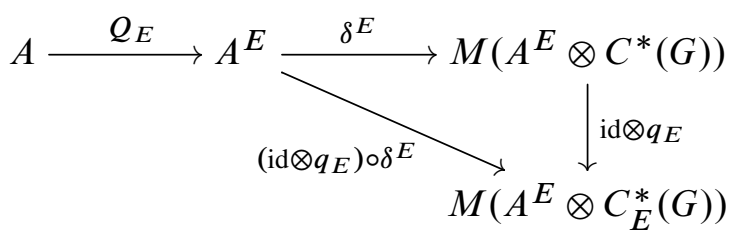

need not be faithful. Thus we cannot characterize the $E$-coactions as the coactions that are " $E$-normal" in the sense that the map $Q_{E}$ is faithful. Furthermore, unlike with normalization, Remark 6.15 shows that $\tau_{E}$ is not isomorphic to its composition with maximalization.

Question 6.19. Let $\mathcal{F}$ be a collection of large ideals of $B(G)$, and let

$$
F=\bigcap_{E \in \mathcal{F}} E .
$$

Then $F$ is a large ideal of $B(G)$. Is $\tau_{F}$ a greatest lower bound for the coaction functors $\left\{\tau_{E}: E \in \mathcal{F}\right\}$ ? (It is easy to see that $\tau_{F}$ is a lower bound.) What if we take $\mathcal{F}$ to be the set of all large ideals $E$ of $B(G)$ for which $\tau_{E}$ is exact?

Question 6.20. Given a coaction functor $\tau$, is there a large ideal $E$ of $B(G)$ such that, after restricting to maximal coactions, $\tau$ is naturally isomorphic to $\tau_{E}$ ? Note that at the level of objects the statement is false: [Buss and Echterhoff 2014, Example 5.4] gives a source of examples of a maximal coaction $(A, \delta)$ and a weakly invariant ideal $I \subset \operatorname{ker} q_{A}^{n}$ such that the quotient coaction $\left(A / I, \delta^{I}\right)$ is not of the form $\left(A^{E}, \delta^{E}\right)$ for any large ideal $E$. (Theorem 6.10 of [KLQ 2016] gives related examples, albeit not involving maximal coactions.)

Here is a related question: do there exist coaction functors that include the BussEchterhoff examples? Such a functor could not be exact, since the Buss-Echterhoff examples are explicitly based upon short exact sequences whose image under the quotient maps are not exact. We could ask the same question for the functor $\tau_{E}$, which, again, is exact for $E=B(G)$ but not for $E=B_{r}(G)$.

Question 6.21. For which large ideals $E$ is the coaction functor $E$-ization exact? Exactness trivially holds for $E=B(G)$, since $B(G)$-ization coincides with maximalization. On the other hand, exactness does not always hold for $E=B_{r}(G)$, because Gromov has shown the existence of nonexact groups. 
Question 6.22. Let $\tau$ be the minimal exact and Morita compatible coaction functor. Applying $\tau$ to the canonical coaction $\left(C^{*}(G), \delta_{G}\right)$, we get a coaction $\left(C^{*}(G)^{\tau}, \delta_{G}^{\tau}\right)$, with a canonical quotient map

$$
q^{\tau}: C^{*}(G) \rightarrow C^{*}(G)^{\tau} .
$$

Then

$$
E_{\tau}:=\left(\operatorname{ker} q^{\tau}\right)^{\perp}
$$

is a large ideal of $B(G)$, by [KLQ 2013, Corollary 3.13].

Does the functor $\tau$ coincide with $E_{\tau}$-ization? This is related to the following question: is

$$
E_{\tau}=\bigcap\{E: E \text { is a large ideal such that } E \text {-ization is exact }\} ?
$$

Again we could ask the analogous questions for $\tau_{E}$. See also the discussion in [Baum et al. 2016, Section 8.1].

Remark 6.23. Related to Question 6.19 above, what if we consider only finitely many large ideals? Let $E$ and $F$ be two large ideals, and let $D=E \cap F$, which is also a large ideal. Suppose that the coaction functors $\tau_{E}$ and $\tau_{F}$ are both exact.

Is $\tau_{D}$ exact? We proved in Corollary 6.9 that exactness of $E$ implies that $D$ is the weak*-closed span of the set of products $E F$, and then we can deduce from this that if

$$
0 \longrightarrow(I, \gamma) \stackrel{\phi}{\longrightarrow}(A, \delta) \stackrel{\psi}{\longrightarrow}(B, \varepsilon) \longrightarrow 0
$$

is a short exact sequence of coactions, and if we assume that $\delta$ is $w$-proper in the sense that $(\omega \otimes \mathrm{id}) \circ \delta(A) \subset C^{*}(G)$ for all $\omega \in A^{*}$, then the sequence

$$
0 \longrightarrow I^{D} \stackrel{\phi^{D}}{\longrightarrow} A^{D} \stackrel{\psi^{D}}{\longrightarrow} B^{D} \longrightarrow 0
$$

is exact. We see a way to parlay this into a proof that $\tau_{D}$ is indeed exact, but this requires a somewhat more elaborate version of Morita compatibility, involving not only imprimitivity bimodules but more general $C^{*}$-correspondences. This will perhaps resemble the property that Buss, Echterhoff and Willett call correspondence functoriality (see [Buss et al. 2015, Theorem 4.9]). We plan to address this in a forthcoming publication.

\section{Appendix: $B(G)$-module lemmas}

Every coaction $\delta: A \rightarrow M\left(A \otimes C^{*}(G)\right)$ gives rise to a $B(G)$-module structure on $A$ via

$$
f \cdot a=(\mathrm{id} \otimes f) \circ \delta(a) \text { for } f \in B(G), a \in A .
$$


We feel that this module structure is under-appreciated, and will point out here several situations in which it makes things easier, since it allows us to avoid computations with tensor products.

Proposition A.1. Let $(A, \delta)$ and $(B, \varepsilon)$ be coactions of $G$, and let $\phi: A \rightarrow B$ be a homomorphism. Then $\phi$ is $\delta-\varepsilon$ equivariant if and only if it is a module map, i.e.,

$$
\phi(f \cdot a)=f \cdot \phi(a) \quad \text { for all } f \in B(G), a \in A \text {. }
$$

Proof. First assume that $\phi$ is $\delta-\varepsilon$ equivariant, and let $f \in B(G)$ and $a \in A$. Then

$$
\begin{aligned}
\phi(f \cdot a) & =\phi((\mathrm{id} \otimes f) \circ \delta(a)) \\
& =(\mathrm{id} \otimes f)((\phi \otimes \mathrm{id}) \circ \delta(a)) \\
& =(\mathrm{id} \otimes f)(\varepsilon \circ \phi(a)) \\
& =f \cdot \phi(a) .
\end{aligned}
$$

Conversely, assume that $\phi$ is a module map, and let $a \in A$. Then for every $f \in B(G)$ the above computation shows that

$$
(\mathrm{id} \otimes f)((\phi \otimes \mathrm{id}) \circ \delta(a))=(\mathrm{id} \otimes f)(\varepsilon \circ \phi(a)),
$$

and it follows that $(\phi \otimes \mathrm{id}) \circ \delta(a)=\varepsilon \circ \phi(a)$ since slicing by $B(G)=C^{*}(G)^{*}$ separates points of $M\left(B \otimes C^{*}(G)\right)$.

Proposition A.2. Let $(A, \delta)$ be a coaction, and let I be an ideal of $A$. Then I is weakly $\delta$-invariant if and only if it is invariant for the module structure, i.e.,

$$
B(G) \cdot I \subset I .
$$

Proof. First assume that $I$ is $\delta$-invariant, and let $f \in B(G)$ and $a \in I$. We must show that $f \cdot a \in I$. Let $q: A \rightarrow A / I$ be the quotient map. We have

$$
\begin{aligned}
q(f \cdot a) & =q((\mathrm{id} \otimes f)(\delta(a))) \\
& =(\mathrm{id} \otimes f)((q \otimes \mathrm{id}) \circ \delta(a)) \\
& =0 \quad(\text { since } I \subset \operatorname{ker}(q \otimes \mathrm{id}) \circ \delta) .
\end{aligned}
$$

Conversely, assume that $I$ is $B(G)$-invariant, and let $a \in I$. We need to show that $a \in \operatorname{ker}(q \otimes \mathrm{id}) \circ \delta$. For every $f \in B(G)$ we have $f \cdot a \in I$, so

$$
0=q(f \cdot a)=(\mathrm{id} \otimes f)((q \otimes \mathrm{id}) \circ \delta(a)) .
$$

It then follows that $(q \otimes \mathrm{id}) \circ \delta(a)=0$ since slicing by $B(G)$ separates points in $M\left(A \otimes C^{*}(G)\right)$. 
Remark A.3. It has been noticed elsewhere in the literature that the $B(G)$-module structure can be useful in other ways. For example, $\delta$ is slice-proper [KLQ 2016, Definition 5.1] if and only if the maps

$$
f \mapsto f \cdot a: B(G) \rightarrow A
$$

are weak*-weak continuous (for $a \in A$ ) [KLQ 2016, Lemma 5.3]. Also, for any full coaction $(A, \delta)$,

$$
A_{0}:=\overline{\operatorname{span}}\{A(G) \cdot A\}
$$

is a $C^{*}$-subalgebra and a nondegenerate $A(G)$-submodule of $A$, where $A(G)$ is the Fourier algebra of $A$, and $\delta$ is nondegenerate if and only if $A_{0}=A$ [Quigg 1994, Lemma 1.2, Corollary 1.5] (see also [Katayama 1984, Lemma 2]). In the same vein, [Quigg 1994, Corollary 1.7] says that if $B$ is a nondegenerate $A(G)$-submodule of $M(A)$, then $\left.\delta\right|_{B}$ is a nondegenerate coaction of $G$ on $B$.

\section{Acknowledgement}

We thank the referee for comments that significantly improved our paper.

\section{References}

[Baum et al. 2016] P. Baum, E. Guentner, and R. Willett, "Expanders, exact crossed products, and the Baum-Connes conjecture”, Ann. K-Theory 1:2 (2016), 155-208. Zbl 1331.46064

[Bédos et al. 2011] E. Bédos, S. Kaliszewski, and J. Quigg, "Reflective-coreflective equivalence", Theory Appl. Categ. 25:6 (2011), 142-179. MR 2805748 Zbl 1232.18002

[Brown and Guentner 2013] N. P. Brown and E. P. Guentner, "New C*-completions of discrete groups and related spaces", Bull. Lond. Math. Soc. 45:6 (2013), 1181-1193. MR 3138486 Zbl 06237632

[Buss and Echterhoff 2014] A. Buss and S. Echterhoff, "Universal and exotic generalized fixed-point algebras for weakly proper actions and duality”, Indiana Univ. Math. J. 63:6 (2014), 1659-1701. MR 3298718 Zbl 1320.46052

[Buss and Echterhoff 2015] A. Buss and S. Echterhoff, "Imprimitivity theorems for weakly proper actions of locally compact groups", Ergodic Theory Dynam. Systems 35:8 (2015), 2412-2457. MR 3456601 Zbl 06540094

[Buss et al. 2015] A. Buss, S. Echterhoff, and R. Willett, "Exotic crossed products and the BaumConnes conjecture", J. Reine Angew. Math. (online publication October 2015).

[Echterhoff and Raeburn 1995] S. Echterhoff and I. Raeburn, "Multipliers of imprimitivity bimodules and Morita equivalence of crossed products", Math. Scand. 76:2 (1995), 289-309. MR 1354585 Zbl 0843.46049

[Echterhoff et al. 2004] S. Echterhoff, S. Kaliszewski, and J. Quigg, "Maximal coactions", Internat. J. Math. 15:1 (2004), 47-61. MR 2039211 Zbl 1052.46051

[Echterhoff et al. 2006] S. Echterhoff, S. Kaliszewski, J. Quigg, and I. Raeburn, A categorical approach to imprimitivity theorems for $C^{*}$-dynamical systems, Mem. Amer. Math. Soc. 850, Providence, RI, 2006. MR 2203930 Zbl 1097.46042

[Fischer 2004] R. Fischer, "Maximal coactions of quantum groups", Preprint 350, University of Münster, SFB 478 Geometrische Strukturen in der Mathematik, 2004. 
[Green 1978] P. Green, "The local structure of twisted covariance algebras", Acta Math. 140:1 (1978), 191-250. MR 0493349 Zbl 0407.46053

[Kaliszewski and Quigg 2009] S. Kaliszewski and J. Quigg, "Categorical Landstad duality for actions", Indiana Univ. Math. J. 58:1 (2009), 415-441. MR 2504419 Zbl 1175.46060

[Katayama 1984] Y. Katayama, "Takesaki's duality for a nondegenerate co-action", Math. Scand. 55:1 (1984), 141-151. MR 769030 Zbl 0598.46042

[KLQ 2013] S. Kaliszewski, M. B. Landstad, and J. Quigg, "Exotic group $C^{*}$-algebras in noncommutative duality", New York J. Math. 19 (2013), 689-711. MR 3141810 Zbl 1294.46047

[KLQ 2016] S. Kaliszewski, M. B. Landstad, and J. Quigg, "Exotic coactions", Proc. Edinburgh Math. Soc. (2) 59:2 (2016), 411-434. Zbl 06580770

[Landstad et al. 1987] M. B. Landstad, J. Phillips, I. Raeburn, and C. E. Sutherland, "Representations of crossed products by coactions and principal bundles", Trans. Amer. Math. Soc. 299:2 (1987), 747-784. MR 869232 Zbl 0722.46031

[Nilsen 1999] M. Nilsen, "Full crossed products by coactions, $C_{0}(X)$-algebras and $C^{*}$-bundles", Bull. London Math. Soc. 31:5 (1999), 556-568. MR 1703865 Zbl 0987.46046

[Quigg 1994] J. C. Quigg, "Full and reduced $C^{*}$-coactions", Math. Proc. Cambridge Philos. Soc. 116:3 (1994), 435-450. MR 1291751 Zbl 0830.22005

Received June 6, 2015. Revised March 31, 2016.

S. KALISZEWSKI

SCHOOL of Mathematical and Statistical SCIENCES

ARIZONA STATE UNIVERSITY

TEMPE, AZ 85287

UNITED STATES

kaliszewski@asu.edu

MAgnus B. LANDSTAD

DEPARTMENT OF MATHEMATICAL SCIENCES

NORWEGIAN UNIVERSITY OF SCIENCE AND TECHNOLOGY

NO-7491 TRONDHEIM

NORWAY

magnusla@math.ntnu.no

JOHN QUIGG

School of Mathematical and Statistical SCIENCES

ARIZONA STATE UNIVERSITY

TEMPE, AZ 85287

UNITED STATES

quigg@asu.edu 


\title{
PACIFIC JOURNAL OF MATHEMATICS
}

Founded in 1951 by E. F. Beckenbach (1906-1982) and F. Wolf (1904-1989)

$$
\text { msp.org/pjm }
$$

\section{EDITORS}

\author{
Don Blasius (Managing Editor) \\ Department of Mathematics \\ University of California \\ Los Angeles, CA 90095-1555 \\ blasius@math.ucla.edu
}

\author{
Paul Balmer \\ Department of Mathematics \\ University of California \\ Los Angeles, CA 90095-1555 \\ balmer@math.ucla.edu \\ Robert Finn \\ Department of Mathematics \\ Stanford University \\ Stanford, CA 94305-2125 \\ finn@math.stanford.edu \\ Sorin Popa \\ Department of Mathematics \\ University of California \\ Los Angeles, CA 90095-1555 \\ popa@math.ucla.edu
}

\author{
Vyjayanthi Chari \\ Department of Mathematics \\ University of California \\ Riverside, CA 92521-0135 \\ chari@math.ucr.edu \\ Kefeng Liu \\ Department of Mathematics \\ University of California \\ Los Angeles, CA 90095-1555 \\ liu@math.ucla.edu \\ Igor Pak \\ Department of Mathematics \\ University of California \\ Los Angeles, CA 90095-1555 \\ pak.pjm@gmail.com \\ Paul Yang \\ Department of Mathematics \\ Princeton University \\ Princeton NJ 08544-1000 \\ yang@math.princeton.edu
}

\section{PRODUCTION}

Silvio Levy, Scientific Editor, production@msp.org

\section{SUPPORTING INSTITUTIONS}

ACADEMIA SINICA, TAIPEI

CALIFORNIA INST. OF TECHNOLOGY

STANFORD UNIVERSITY

UNIV. OF BRITISH COLUMBIA

UNIV. OF CALIFORNIA, BERKELEY

UNIV. OF CALIFORNIA, DAVIS

UNIV. OF CALIFORNIA, LOS ANGELES

UNIV. OF CALIFORNIA, RIVERSIDE

UNIV. OF CALIFORNIA, SAN DIEGO

UNIV. OF CALIF., SANTA BARBARA
KEIO UNIVERSITY

MATH. SCIENCES RESEARCH INSTITUTE

NEW MEXICO STATE UNIV.

OREGON STATE UNIV.
Daryl Cooper

Department of Mathematics

University of California

Santa Barbara, CA 93106-3080 cooper@math.ucsb.edu

Jiang-Hua Lu

Department of Mathematics

The University of Hong Kong

Pokfulam Rd., Hong Kong

jhlu@maths.hku.hk

$$
\text { Jie Qing }
$$

Department of Mathematics

University of California

Santa Cruz, CA 95064

qing@ cats.ucsc.edu

\author{
UNIV. OF CALIF., SANTA CRUZ \\ UNIV. OF MONTANA \\ UNIV. OF OREGON \\ UNIV. OF SOUTHERN CALIFORNIA \\ UNIV. OF UTAH \\ UNIV. OF WASHINGTON \\ WASHINGTON STATE UNIVERSITY
}

These supporting institutions contribute to the cost of publication of this Journal, but they are not owners or publishers and have no responsibility for its contents or policies.

See inside back cover or msp.org/pjm for submission instructions.

The subscription price for 2016 is US $\$ 440 /$ year for the electronic version, and \$600/year for print and electronic.

Subscriptions, requests for back issues and changes of subscriber address should be sent to Pacific Journal of Mathematics, P.O. Box 4163, Berkeley, CA 94704-0163, U.S.A. The Pacific Journal of Mathematics is indexed by Mathematical Reviews, Zentralblatt MATH, PASCAL CNRS Index, Referativnyi Zhurnal, Current Mathematical Publications and Web of Knowledge (Science Citation Index).

The Pacific Journal of Mathematics (ISSN 0030-8730) at the University of California, c/o Department of Mathematics, 798 Evans Hall \#3840, Berkeley, CA 94720-3840, is published twelve times a year. Periodical rate postage paid at Berkeley, CA 94704, and additional mailing offices. POSTMASTER: send address changes to Pacific Journal of Mathematics, P.O. Box 4163, Berkeley, CA 94704-0163.

PJM peer review and production are managed by EditFLOW ${ }^{\circledR}$ from Mathematical Sciences Publishers.

PUBLISHED BY

\section{I. mathematical sciences publishers}

nonprofit scientific publishing

http://msp.org/

(C) 2016 Mathematical Sciences Publishers 


\section{PACIFIC JOURNAL OF MATHEMATICS}

Volume $284 \quad$ No. $1 \quad$ September 2016

Bitwist manifolds and two-bridge knots

JAmes W. CANNON, William J. Floyd, LEeR LAMbert,

WALTER R. PARry and Jessica S. PurCELL

Recognizing right-angled Coxeter groups using involutions

Charles Cunningham, Andy Eisenberg, Adam Piggott and KIM RUANE

On Yamabe-type problems on Riemannian manifolds with boundary

Marco Ghimenti, Anna Maria Micheletti and Angela

PISTOIA

Quantifying separability in virtually special groups

MARK F. HAGEN and PRIYAM PATEL

Conformal designs and minimal conformal weight spaces of vertex operator superalgebras

TOMONORI HASHIKAWA

Coaction functors

S. KALiszewski, Magnus B. LANDSTAD and John QuigG

Cohomology and extensions of braces

VICTORIA LEBED and LEANDRO VENDRAMIN

Noncommutative differentials on Poisson-Lie groups and pre-Lie algebras

SHAHN MAJID and WEN-QING TAO 